\title{
Identifying Potential Diagnostic Genes for Diabetic Nephropathy Based on Hypoxia and Immune Status
}

\author{
Changyan $\mathrm{Li}^{\prime}$ \\ Feng Su' \\ Le Zhang ${ }^{2}$ \\ Fang $\mathrm{Liu}^{3}$ \\ Wenxing Fan (ID) \\ Zhen $\mathrm{Li}^{4}$ \\ JingYuan $\mathrm{Ma}^{\mathrm{I}}$
}

'Department of Nephrology, the First Affiliated Hospital of Kunming Medical University, Kunming, Yunnan Province, People's Republic of China; ${ }^{2}$ Institute for Integrative Genome Biology, University of California Riverside, Riverside, CA, USA;

${ }^{3}$ Department of Nephrology, West China Hospital, Sichuan University, Chengdu, Sichuan Province, People's Republic of China; ${ }^{4}$ Organ Transplantation Center, the First Affiliated Hospital of Kunming Medical University, Kunming, Yunnan Province, People's Republic of China
Correspondence: Wenxing Fan Email fanwx2020@163.com
Background: The prognosis of diabetic nephropathy is poor, and early diagnosis of diabetic nephropathy is challenging. Fortunately, searching for DN-specific markers based on machine algorithms can facilitate diagnosis.

Methods: xCell model and CIBERSORT algorithm were used to analyze the relationship between immune cells and DN, and WGCNA analysis was used to evaluate the regulatory relationship between hypoxia gene and DN-related immune cells. Lasso regression and ROC regression were used to detect the ability of core genes to diagnose DN, the PPI network of core genes with high diagnostic ability was constructed, and the interaction between core genes was discussed.

Results: There were 519 differentially expressed genes in renal tubules and 493 differentially expressed genes in glomeruli. Immune and hypoxia responses are involved in the regulation of renal glomerulus and renal tubules. We found that there are 16 hypoxia-related genes involved in the regulation of hypoxia response. Seventeen hypoxia-related genes in renal tubules are involved in regulating hypoxia response on the proteasome signal pathway. Lasso and ROC regression were used to screen anoxic core genes. Further, we found that TGFBR3, APOLD1, CPEB1, and KDR are important in diagnosing DN glomerulopathy, respectively, PSMB8, PSMB9, RHOA, VCAM1, and CDKN1B, which have high specificity for renal tubulopathy in DN.

Conclusion: Hypoxia and immune reactions are involved in the progression of DN. T cells are the central immune response cells. TGFBR3, APOLD1, CPEB1, and KDR have higher diagnostic accuracy in the diagnosis of DN. PSMB8, PSMB9, RHOA, VCAM1, and CDKN1B have higher diagnostic accuracy in DN diagnosis.

Keywords: diabetic nephropathy, early diagnosis, LASSO model, T cells, WGCNA, hypoxia

\section{Introduction}

Diabetic nephropathy (DN) is a chronic vascular complication of diabetes, accounting for $40-50 \%$ of diabetic patients, ${ }^{1}$ with a large population base, easy to develop into proteinuria and terminal stage, poor prognosis. At present, the pathogenesis of $\mathrm{DN}$ is not clear, so it is necessary to study the mechanism.

Glucose utilization in diabetes is impaired. ${ }^{2}$ With the increase of glucose in the urinary system, mesangial cells and podocytes are exposed to high glucose, and the inhibition of aerobic metabolism leads to cell damage in a hypoxic environment; this leads to the damage to the basement membrane and the exposure of protein and 
other macromolecular substances, and the formation of proteinuria. ${ }^{3}$ For example, recent studies have shown that hypoxia is a critical factor in tubular atrophy and renal interstitial fibrosis. ${ }^{4}$ In addition, hypoxia or ischemic stress can also affect various kidney diseases, such as renal cortex or medulla hypoxia can lead to tissue damage and cause acute and chronic kidney disease development. ${ }^{5-7}$ In kidney disease hypoxia, the research found that the glomerulus and renal tubular injury have different regulatory roles, suggesting that hypoxia is emerging as a new renal lesion mechanism. In addition, a large number of studies have confirmed that immunity is the crucial factor of kidney disease. ${ }^{8-10}$ Based on the existing studies of hypoxia immunity in kidney disease, this study constructed the diagnosis model of hypoxia and immune staterelated DN, and it is expected to be used in the early clinical diagnosis of DN shortly. At the same time, studies have shown that almost all kidney diseases exhibit cytokine expression and infiltration of immune cells, suggesting that the kidney targets the immune response.

Moreover, hypoxia is now being considered as a potential immunomodulator. In cancer research, the relationship between hypoxia and the regulation of immune function has been proved. There is potential value in investigating the potential diagnostic genes for DN based on the correlation between hypoxia and the immune response.

As a clinical diagnosis, clinical studies have shown that renal biopsy is the gold standard for diagnosing renal diseases. ${ }^{11,12}$ However, there are few clinical applications for early diagnosis as an invasive procedure, ${ }^{13}$ making early diagnosis difficult. The current noninvasive diagnosis lacks creatinine sensitivity. Massive proteinuria and end-stage proteinuria account for the vast majority of DN. The prognosis is very limited. ${ }^{14}$ This study explores the potential diagnostic genes for DN based on the correlation between hypoxia and immune response to address this dilemma to some extent. We believe that the hypoxia diagnostic gene is expected to be used as an early diagnostic tool for DN. It is beneficial to the early diagnosis of DN. Studies have shown that early diagnosis can help to improve patients' quality of life. ${ }^{15,16}$ In addition, based on the gene expression profiling data of $\mathrm{DN}$, we designed to study the molecular changes and biological mechanisms of $\mathrm{DN}$-induced glomerular and tubular lesions. In order to early diagnose DN patients, we used the combination of the LASSO model and ROC curve regression (ROC) to predict the specific diagnostic gene of $\mathrm{DN}$ and validated it with external data to identify the early diagnostic molecule of DN.

\section{Materials and Methods Data Collection}

The study was based on data from the http://www.ncbi. nlm.nih.gov/go/ Database provided by the public dataset. Besides, GSE30122 data ${ }^{17}$ as the experimental data set, in which 9 cases of DN patients with glomerular specimens and 26 normal controls of glomerular specimens, there are 24 normal controls of renal tubular, and the $10 \mathrm{DN}$ patients were all renal biopsy specimens. In addition, the GSE96804 data ${ }^{18}$ was used as the validation data set, including glomerular tissues of $41 \mathrm{DN}$ patients and 20 normal controls, including glomerular tissues of $41 \mathrm{DN}$ patients and 20 normal controls. The control group met the following criteria: estimated renal function above $90 \mathrm{~mL} /$ min, no proteinuria, normal serum creatinine, urea nitrogen. According to clinical and pathological features, DN patients were divided into early DN Group $(n=20$, EGFR $>60 \mathrm{~mL} / \mathrm{min}$, glomeruli classified as class I or IIA) and late DN Group $(\mathrm{N}=21$, EGFR between $15 \mathrm{~mL} / \mathrm{min}$ and $60 \mathrm{~mL} / \mathrm{min}$, glomeruli classified as class III or IV). In addition, in the GSE30529 dataset, ${ }^{17} 10$ renal tubular specimens were from DN patients and 12 normal controls. Kidney specimens GSE30529 and GSE30122 were obtained from living donor renal allografts, surgical nephrectomy, and remnants of a diagnostic renal biopsy, respectively. As for the GSE142025 database, ${ }^{19}$ from January 2015 to December 2016, a total of 28 biopsied diabetic nephropathy patients were admitted to the sixth People's Hospital affiliated to Shanghai Jiao Tong University. According to the guidelines of the local ethics committee, kidney tissue is collected through ultrasoundguided kidney biopsies with informed consent. The samples were early $\mathrm{DN}(\mathrm{N}=6)$ and late $\mathrm{DN}(\mathrm{n}=22)$. The early DN was defined as UACR ranging from 30 to $300 \mathrm{mg} / \mathrm{g}$, EGFR as $90 \mathrm{~mL} / \mathrm{min} / 1.73 \mathrm{~m}^{2}$, and the early $\mathrm{DN}$ as UACR $300 \mathrm{mg} / \mathrm{g}$, EGFR $90 \mathrm{~mL} / \mathrm{min} / 1.73 \mathrm{~m}^{2}$.

\section{Analysis of Differentially Expressed Genes in DN}

Firstly, gene expression profiles of DN group and control group were established. Then, in the GSE30122, GSE96804 and GSE30529 gene expression data sets, the differences between DN Group and control group were analyzed by using $\mathrm{R}$ (version 4.0.3, X64) software, 
"Limma" $\mathrm{R}$ package, and the screening conditions were set as $\operatorname{LogFC}>1, P<0.05$.

\section{GO Enrichment and KEGG Pathway Analysis}

GO is a database jointly established by gene ontology to screen protein groups in $\mathrm{GO}$ annotation based on $P<0.05$, the purpose of this study is to establish a semantic lexical standard that can be applied to various species and to define and describe the significance of biological functions of genes and proteins with $P$ values. GO can be divided into three categories: molecular functional (MF), biological process (BP) and cell component (CC), through which the functions of genes can be defined and described in a variety of ways. Based on the annotation co-degree, the annotated terms are clustered, and the cluster values are given by using the modulus and clustering algorithm. The higher the score, the more prominent the gene is on the gene list. In addition, KEGG is an integrated database that integrates genomic, chemical and systemic functional information on the genetic pathways of different species. Using R cluster Profiler Package "org.Hs.eg.db". Protein functional groups in KEGG annotation were screened by using P-value $(P<0.05)$, respectively. Based on the enrichment analysis of serviceable items, the potential targets of autophagy genes were investigated for their biological functions and involved biological pathways. In our study, GO enrichment analysis and KEGG pathway analysis and visualization were performed utilizing $\mathrm{R}$. It was considered a significant difference in $P<0.05$.

\section{Co-Expression Analysis}

Based on the results of differential analysis of glomerular and tubular gene expression of DN patients with GSE30122 database, we further used weighted coexpression network analysis (WGCNA). The coexpression network of DN associated DEGs in GSE30122 was determined. Using R (version 4.0.3, X64) software. WGCNA package was installed to analyze the co-expression of differentially expressed genes. In the calculation, we use multi-thread work, set the power function index range to $1: 20$, calculate the dependency matrix: "ai, $j=|\operatorname{Cor}(i, J)| \beta$ ", determine the best $\beta$ value, and then calculate the module gene number according to the similarity between two genes by topological overlap (TOM), then the hierarchical clustering analysis is carried out, through the transformation of the dissimilarity between genes, the gene clustering tree is obtained, and the dynamic hybrid cutting algorithm is used to cut the gene clustering tree, and the gene number of each module is set to 50 , so we can divide the identical genes into the same modules. Finally, we merge very similar modules through module exigencies (ME).

\section{LASSO and ROC Regression}

For the diabetic nephropathy genes related to hypoxia, we used LASSO regression analysis, R (version 4.0.3, X64) software, installed "planet" R software package, set "alpha $=1$ ", set "Family = binomial" to the binary variable of this study, the training and validation set ratio was 3:7, with the optimal $\lambda$ value was calculated. Core genes for the ability of differential genes to diagnose DN were screened. Besides, core genes obtained from LASSO regression screening of GSE30122, GSE96804 and GSE30529 datasets were analyzed by ROC regression analysis using "pROC" R software package. The AUC gene with a mean value greater than 0.6 was identified as the key gene using "ggplot2" R software package.

\section{Correlation Between Immune Cell Status and DN-Related Differentially Expressed Genes}

The correlation between DEGs of DN and immune cells was analyzed using the CIBERSORT algorithm (http:// cibersort.stanford.edu/); it is a method of correlation calculation based on the LM22 dataset, LM22 is an approach to accurately differentiate 22 human hematopoietic cell phenotypes, including B cells, T cells, natural killer (NK) cells, macrophages, dendritic cells (DCs), and myeloid subsets. A deconvolution algorithm published by Alizadeh et al quantifies the cellular components of a rich gene expression profiling - using the CIBERSORT algorithm to evaluate the relative fraction of DN and 22 kinds of immune cells.

Figure 1D shows 22 kinds of immune cells and renal tubular differentially expressed genes. Statistical processing uses the "CIBERSORT" $\mathrm{R}$ package for correlation analysis. Blue indicates a positive correlation between the two variables, and red indicates a negative correlation. The deeper the color, the more significant the correlation of variables; here, the color represents R-value from -1 to +1 , positive correlation, negative correlation; the blue and noon filling charts show a positive correlation between the two variables, while the red and anticlockwise direction 
A

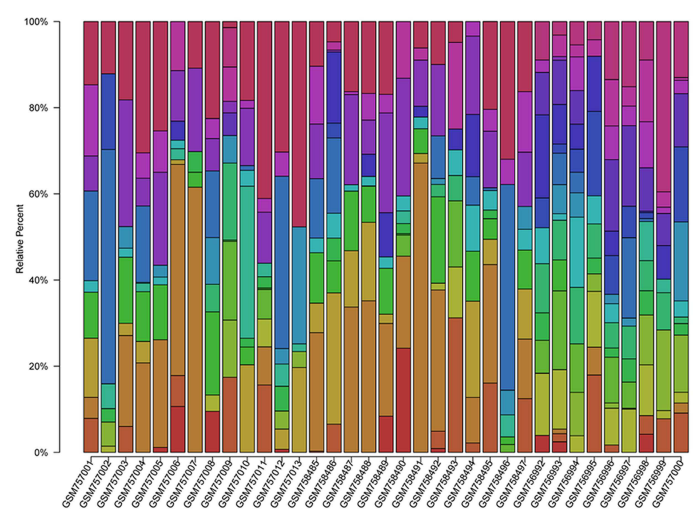

C

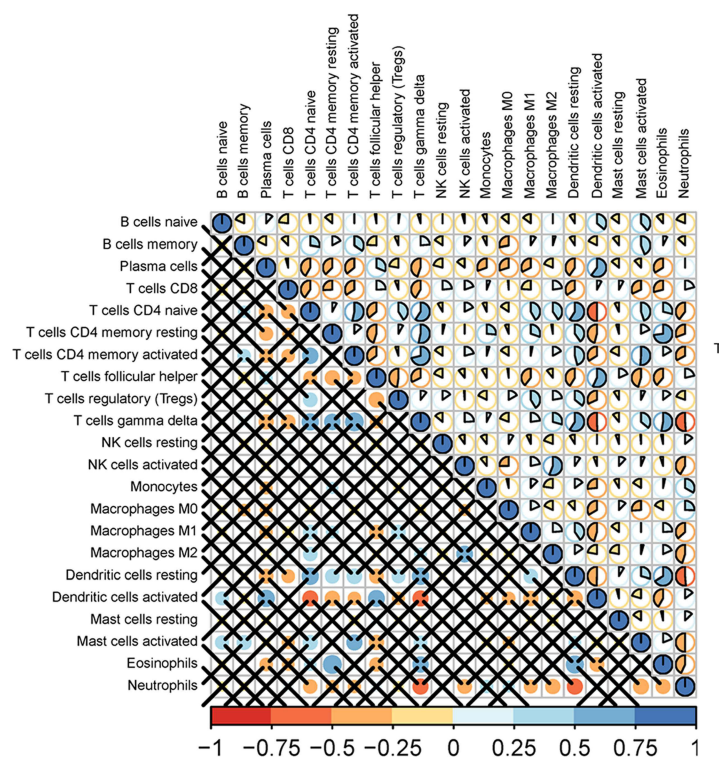

B

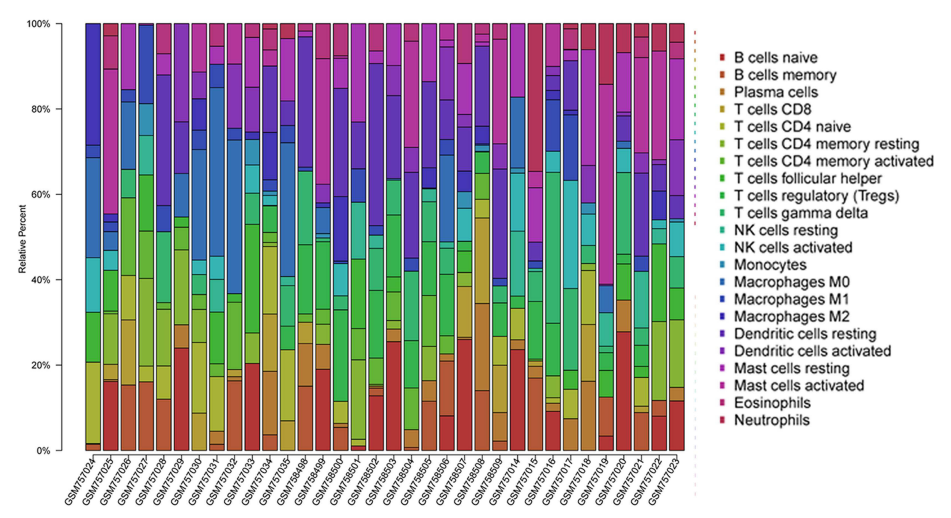

D

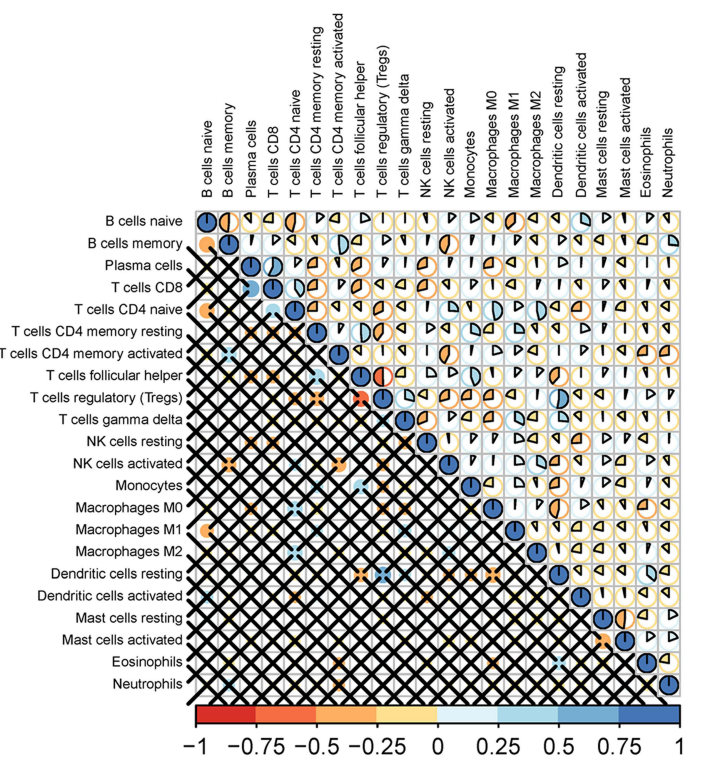

Figure I (A) Immunologic correlation analysis of glomerular tissues in patients with DN; (B) Immunologic correlation analysis of renal tubular tissues in patients with DN; (C) Correlation analysis of differential immunocytes in the glomeruli of patients with DN; (D) For the tubules of patients with DN, with the correlation analysis of differential immunocytes.

charts show a negative correlation. The lower left-hand corner of the correlation coefficient test set $\mathrm{P}<0.05$ for a significant correlation, no statistical significance of the " $\times$ " symbol. The immune and DN, the GSE142025 database, was selected to explore the immune cells and DN relationship. The quantity of DN-related gene expression was obtained by differential analysis using the "limma" package in the R software for the DN transcription data set. It then evaluated the correlation between gene expression and immune cell expression by combining differential gene expression with the xCell (https://xcell.ucsf.edu/) algorithm. xCell is a recently published method based on ss-GSEA that estimates the abundance score of 64 immune cell types, including adaptive and innate immune cells, hematopoietic progenitors, epithelial cells, and extracellular matrix cells. In our study, we used this algorithm to do immune correlation analysis to get the results of immune gene enrichment. Furthermore, in this study Figure 2A, the Y-axis shows the predicted percentage of immune cells in each sample.

\section{PCA Analysis}

Principal component analysis $(\mathrm{PCA})^{20}$ is a method that uses an orthogonal transformation to transform the observed values of a series of variables that may be related linearly and then project the values of a series of linearly unrelated variables to make statistical analysis and simplify the data set. Move the Axis Center to the center of 
A
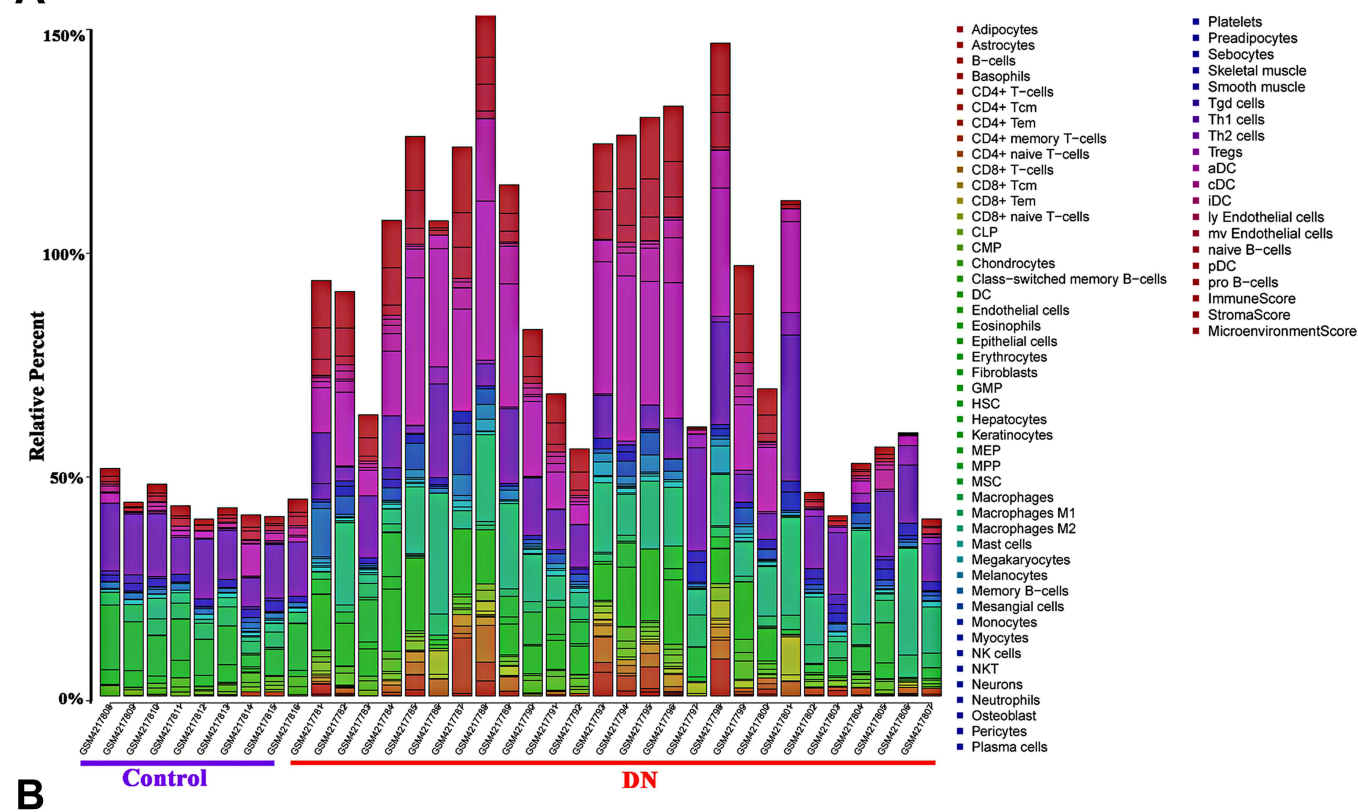

B
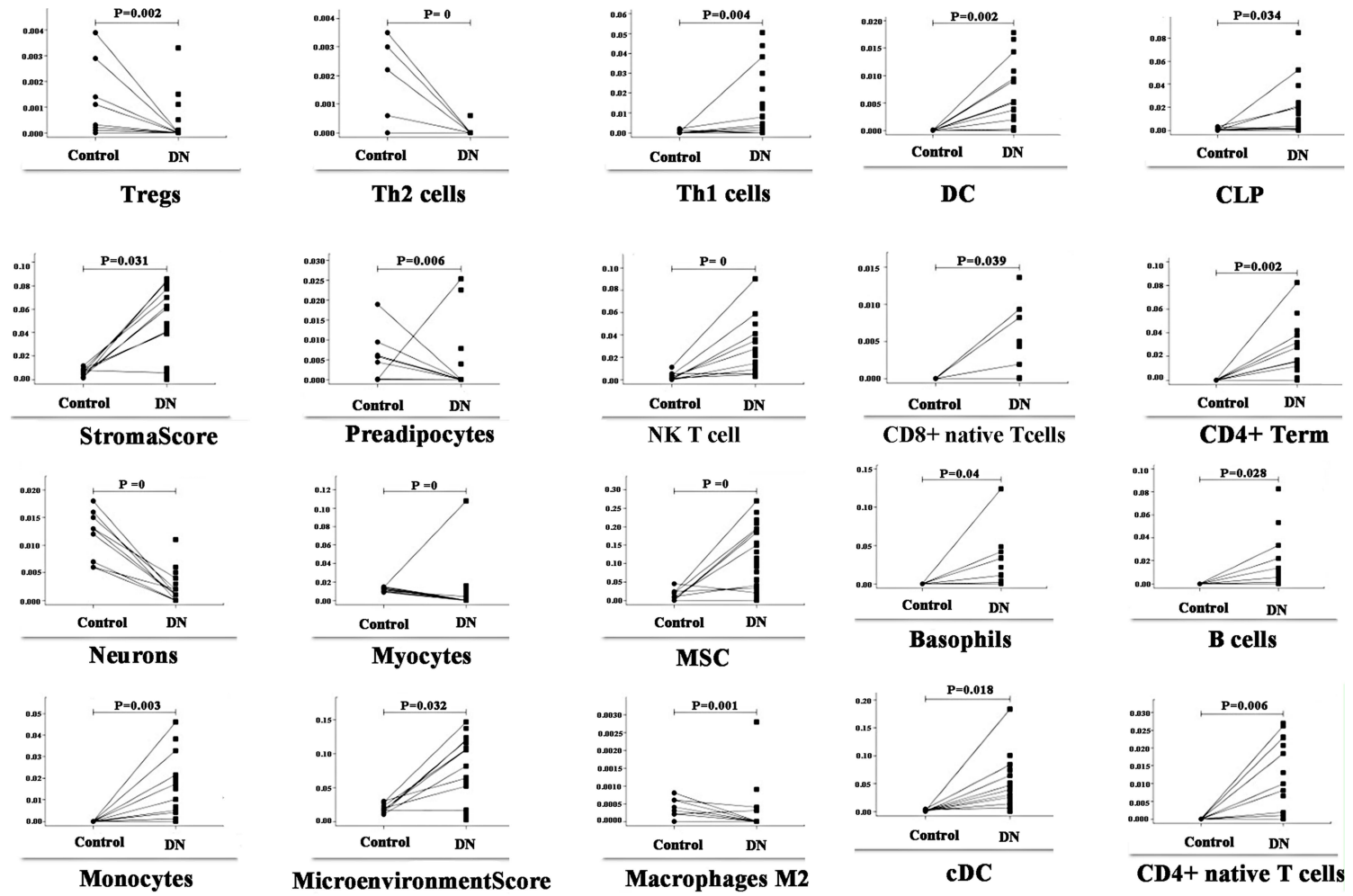

MicroenvironmentScore
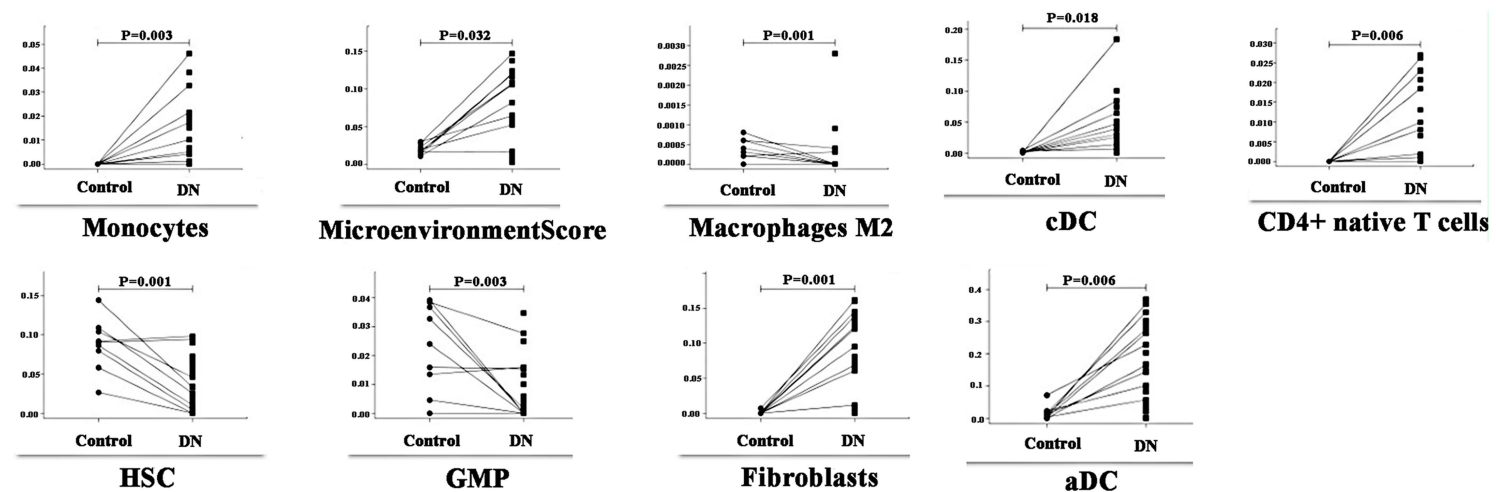

Figure 2 (A) xCell model of DN, kidney tissue data of GSE142025 was based on 64 immunocyte phenotypes to verify the expression of immune cells after DN; (B) Expression of many immune cells after DN. 
the data, and then rotate the axis so that the variance of the data on the $\mathrm{C} 1$ axis is the largest; that is, the projection of all $\mathrm{n}$ data individuals in that direction is the most scattered. This means more information is kept. $\mathrm{C} 1$ becomes the first principal component. C 2-second principal component: find a $\mathrm{C} 2$ with a covariance (correlation coefficient) of 0 between $\mathrm{c} 2$ and $\mathrm{C} 1$ to avoid overlapping with $\mathrm{c} 1$ information and maximize the variance in that direction. In this paper, we use this method to perform $\log 2 \mathrm{~N}$ (normalization) after merging the random GSE99340 and GSE30529 samples and use PCA to visualize the normalized results after data correction and verify the diagnostic ability of DN with the merged standardized data set.

\section{Results}

\section{$x$ Cell Model T Cells Participate in the Regulation of DN}

xCell model was based on 64 immune cell phenotypes to validate the DN association. The results showed that multiple immune cells were significantly upregulated (Figure 2A) after renal injury, with the $\mathrm{T}$ cell subtypes Tregs, Th1, Th2, NKT, CD4 + and CD8 + were significantly different $(P<0.05)$, Tregs, Th2 were significantly down-regulated $(P<0.05)$, Th1 NKT, CD4 + and CD8 + subtypes were significantly upregulated $(P<0.05)$, which indicated that $\mathrm{T}$ cells participated in $\mathrm{DN}$ regulation. Different subtypes showed different changes (Figure 2B). Besides, the study is shown in Figure 3.

\section{Cellular Immunity is Associated with DN}

Glomerulus and renal tubules in the nephron of diabetes mellitus have different degrees of pathological changes after secondary renal disease. Based on the CIBERSORT algorithm, 22 kinds of immune cells changed after DN and tubulopathy were predicted. However, the changes of 22 kinds of immune cells were different, for the immune cells in the DN group were significantly changed $(P<0.05)$. Principal component analysis (PCA) showed significant differences between the immune cells in the DN group and those in the control group. There was a negative correlation between macrophages and various immune cells (Figure 1A and C). In addition, some immune cells in the DN group were different from those in the control group $(P<0.05)$. Most of the immune cells were the same as normal controls, mainly represented by $\mathrm{T}$ cell lines and $\mathrm{B}$ cell lines. Macrophage cell lines were negatively correlated with many immune cells (Figure 1B and D).

\section{Activation of $\mathrm{T}$ Cell Lines in $\mathrm{DN}$ is Associated with Hypoxia}

There were 503 hypoxia-related genes in the human disease spectrum downloaded from the UniProt database. In addition, 519 differentially expressed genes in the renal tubules and 493 differentially expressed genes in the glomerular tissues of the GSE30122 samples were obtained by differential analysis. Veen analysis of hypoxia-related genes and DN differentially expressed genes showed that the total number of hypoxia genes was A (value 503) and the glomerular differentially expressed genes was B (value 493). In patients with $\mathrm{DN}$, the number of hypoxiaassociated genes in the glomerulus is set to $\mathrm{C}$, so the formula for calculating the proportion of hypoxia genes in the glomerulus is $\mathrm{C}=16 / \mathrm{A}+\mathrm{B}^{*} 100 \%$. The results showed no significant difference in the number of genes in the renal tubules and the number of genes in the glomerular tissues of the GSE30122 sample. Using Venn diagram analysis, 17 anoxic genes were found in renal tubular samples associated with $\mathrm{DN}$, which plays an anoxic response and is mainly enriched in the phagocytic signalling pathway (Figure 4A-C). Sixteen hypoxia genes were involved in hypoxia response and enriched in the HIF1 signalling pathway (Figure 4-F) in glomerular samples, which were related to $\mathrm{DN}$.

\section{Hypoxia Differentially Expressed Genes Can Activate Some Immune Cells in the Process of DN}

During the process of DN, the major immune cells of the glomeruli, such as $\mathrm{T}$ cells, $\mathrm{T}$ cell $\mathrm{CD} 8$, $\mathrm{T}$ cell $\mathrm{CD} 4$ subtypes, dendritic cells, eosinophil granulocyte, macrophage subtypes, plasma cells, neutrophil, mast cells, were significantly changed (Figure 5, $P<0.05$ ), and renal tubular cells affected neutrophil, T cells CD4 and natural killer cell (Figure 5, $P<0.05$ ).

\section{Hypoxia-Associated Genes, TGFBR3, APOLDI, CPEBI and KDR, Have High Diagnostic Accuracy in Glomerulopathy of DN}

Four genes associated with hypoxia, TGFBR3, APOLD1, CPEB1, and KDR, were screened by LASSO regression and ROC regression to evaluate the accuracy of diabetes mellitus. The results showed that there were significant changes in TGFBR3, APOLD1, CPEB1, and KDR after DN. For 


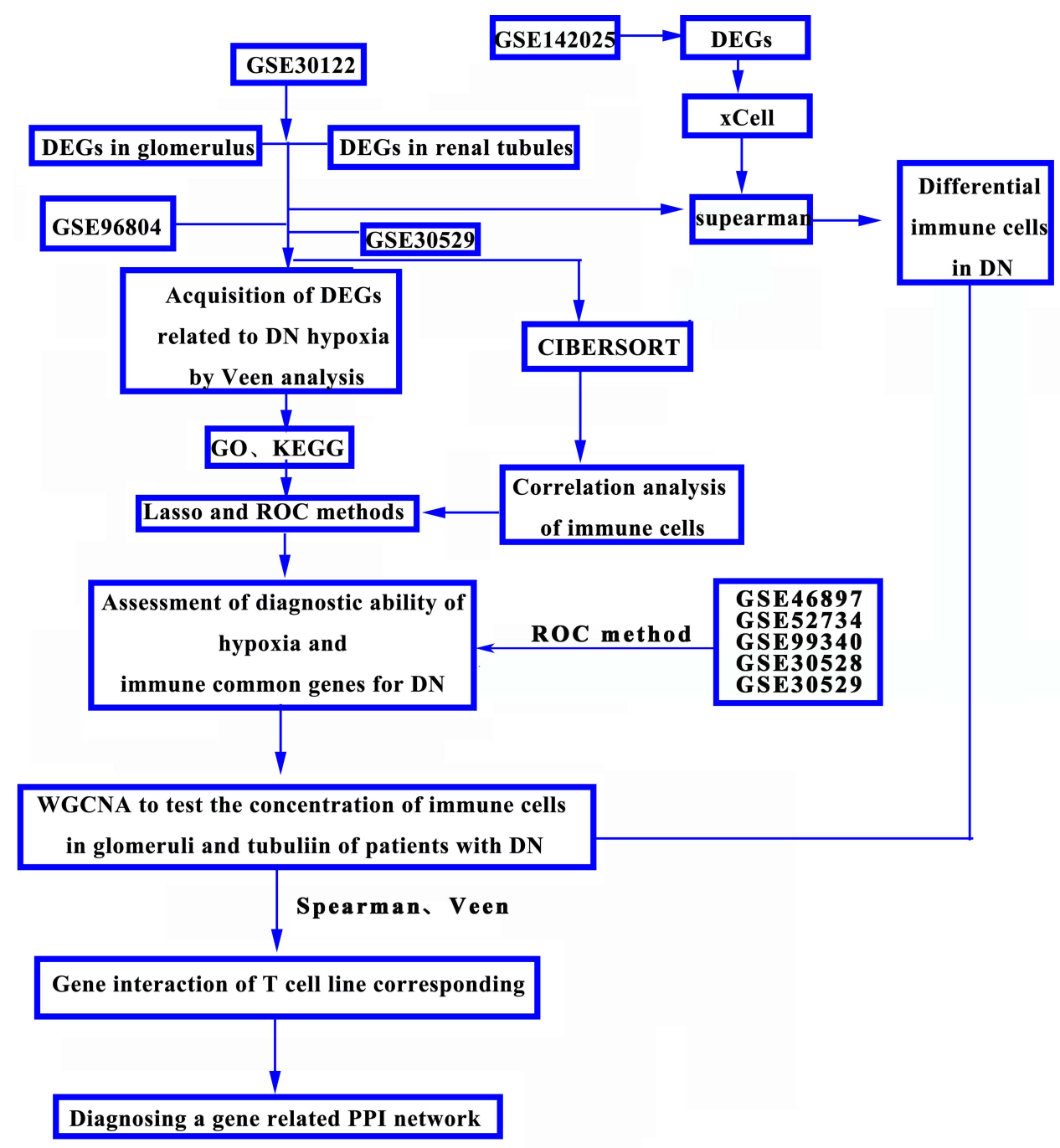

Figure 3 Identification of potential diagnostic biomarkers of DN.

further ROC regression, the diagnostic ability of the four genes was evaluated. In the test GSE30122 dataset, the AUC values of TGFBR3 (0.559), APOLD1 (0.818), CPEB1 (0.661), and KDR (0.514); Besides, to validate the results of a test set, the GSE96804 dataset was used to evaluate the diagnostic ability of glomerulopathy of DN, and it turns out that the AUC values of TGFBR3 (0.915), APOLD1 (0.606), CPEB1(0.676), and KDR (0.818); The results showed that average diagnostic accuracy of these four genes (TGFBR3, APOLD1, CPEB1, and KDR) was $0.737,0.712,0.669$ and 0.666 (Figure 6A-D). Further evidence confirming hypoxia-related genes TGFBR3, APOLD1, CPEB1, and KDR can play a significant diagnosis of glomerulopathy of DN. GSE30528 database also is used to evaluate the diagnostic accuracy by ROC regression; meanwhile, studies have shown that podocyte plays a crucial role in the normal functioning of glomerulus, and its damage will lead to the destruction of glomerulus function. Therefore, we selected diabetic nephropathy podocyte transcriptomic data set GSE46897 to verify the diagnostic ability of TGFBR3, APOLD1, CPEB1, and KDR genes. It is shown that in the glomerular transcriptomic dataset GSE30528, the diagnostic AUC values of TGFBR3, APOLD1, CPEB1, and KDR genes were 1.00, 0.915, 0.983 , and 0.812 (Figure 7A), respectively, and were significantly down-regulated in the DN group (Figure 7B-E), showing the high diagnostic ability for glomerular injury. Meanwhile, in podocyte transcriptomic dataset GSE46897, the DIAGNOSTIC AUC values of TGFBR3, APOLD1, CPEB1, and KDR genes were 0.875, 0.750, 0.625, and 0.625 (Figure 7F), respectively, showing the same high diagnostic ability. In conclusion, TGFBR3, APOLD1, 
A

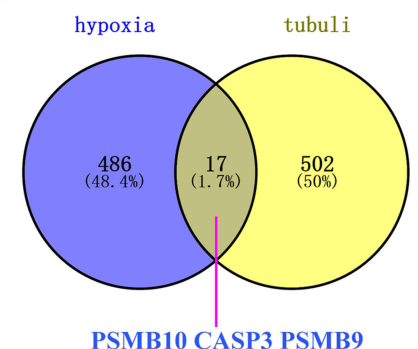

PSMB10 CASP3 PSMB9

PDLIM1 POSTN CDKN1B CAV1

CXCR4 HTR2B HSD11B2 PSMA3 RHOA REG1A COL4A1 VCAM1 PSMB8 MYC
B
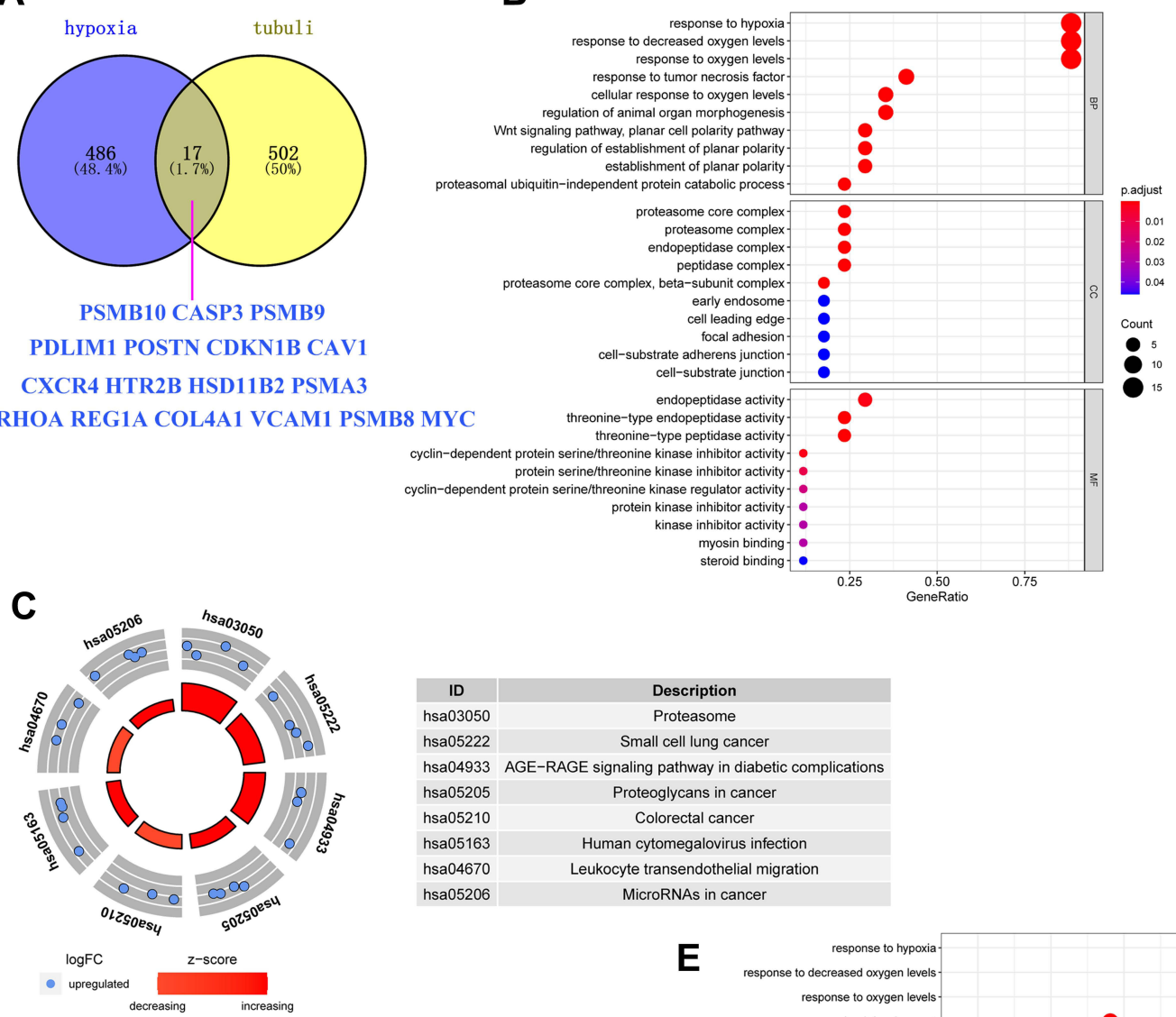

\begin{tabular}{|c|c|}
\hline ID & Description \\
\hline hsa03050 & Proteasome \\
\hline hsa05222 & Small cell lung cancer \\
\hline hsa04933 & AGE-RAGE signaling pathway in diabetic complications \\
\hline hsa05205 & Proteoglycans in cancer \\
\hline hsa05210 & Colorectal cancer \\
\hline hsa05163 & Human cytomegalovirus infection \\
\hline hsa04670 & Leukocyte transendothelial migration \\
\hline hsa05206 & MicroRNAs in cancer \\
\hline
\end{tabular}

\section{E}

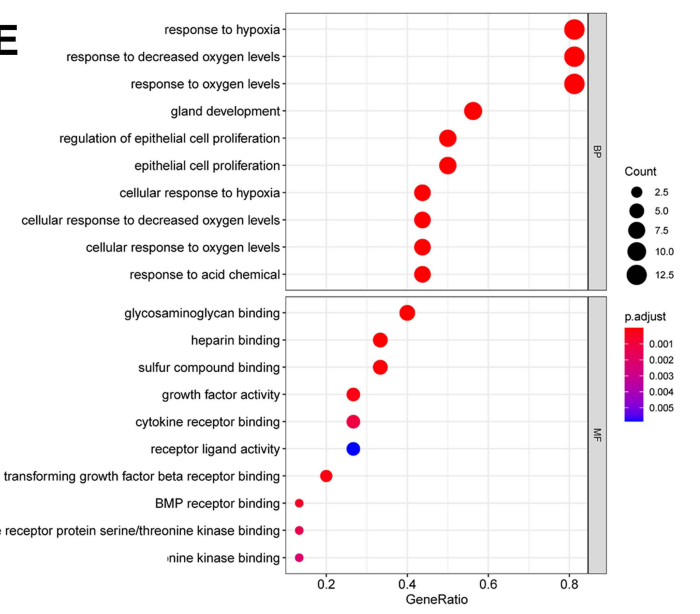

SFRP1

BMP7 BMP2 VEGFA

REG1A NDNF TGFBR3

APOLD1 CPEB1 PTPRB

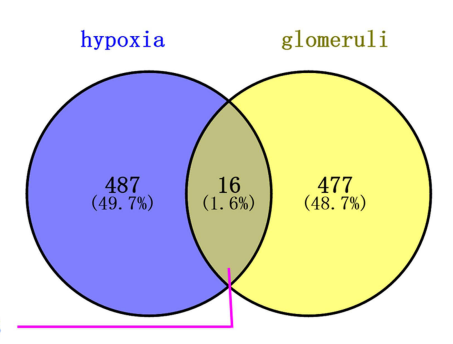

ACTN4 KDR HMOX1 CITED2 TMEM204 CDKN1B

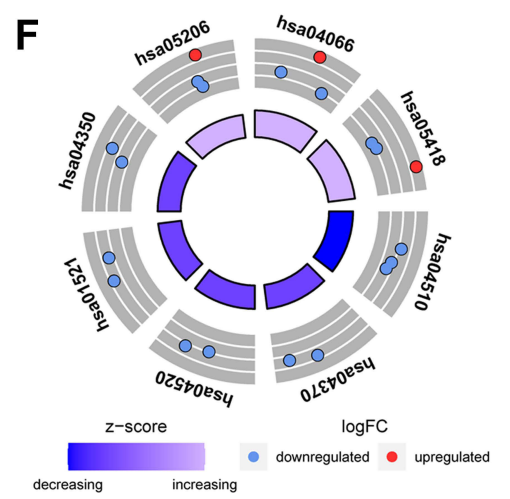

transmembrane receptor protein serine/threonine kinase binding.

\begin{tabular}{|c|c|}
\hline ID & Description \\
\hline hsa04066 & HIF-1 signaling pathway \\
\hline
\end{tabular}

hsa05418 Fluid shear stress and atherosclerosis

hsa04510 Focal adhesion

hsa04370 VEGF signaling pathway

hsa04520 Adherens junction

hsa01521 EGFR tyrosine kinase inhibitor resistance

hsa04350 TGF-beta signaling pathway

hsa05206 MicroRNAs in cancer

Figure 4 (A) Intersection of differential genes in renal tubular tissues and hypoxia gene in human diseases in DN samples from GSE30I22; (B) Enrichment and analysis of renal tubular differential gene and hypoxia intercross gene function; (C) Enrichment analysis of renal tubular differential gene and anoxic cross signal pathway; (D) Genetic Intersection of differential genes in renal globe tissue of DN patients in the GSE30I 22 sample and occurrence of hypoxia gene in human diseases in UniProt database; (E) Enrichment analysis of differential gene and hypoxia intersection signal pathway in renal gullet; (F) Enrichment analysis of renal tubular differential gene and anoxic cross signal pathway in which the HIF-I signal pathway is the main enriched signal pathway of renal tubular differential gene (pink box). 
A

glomeruli
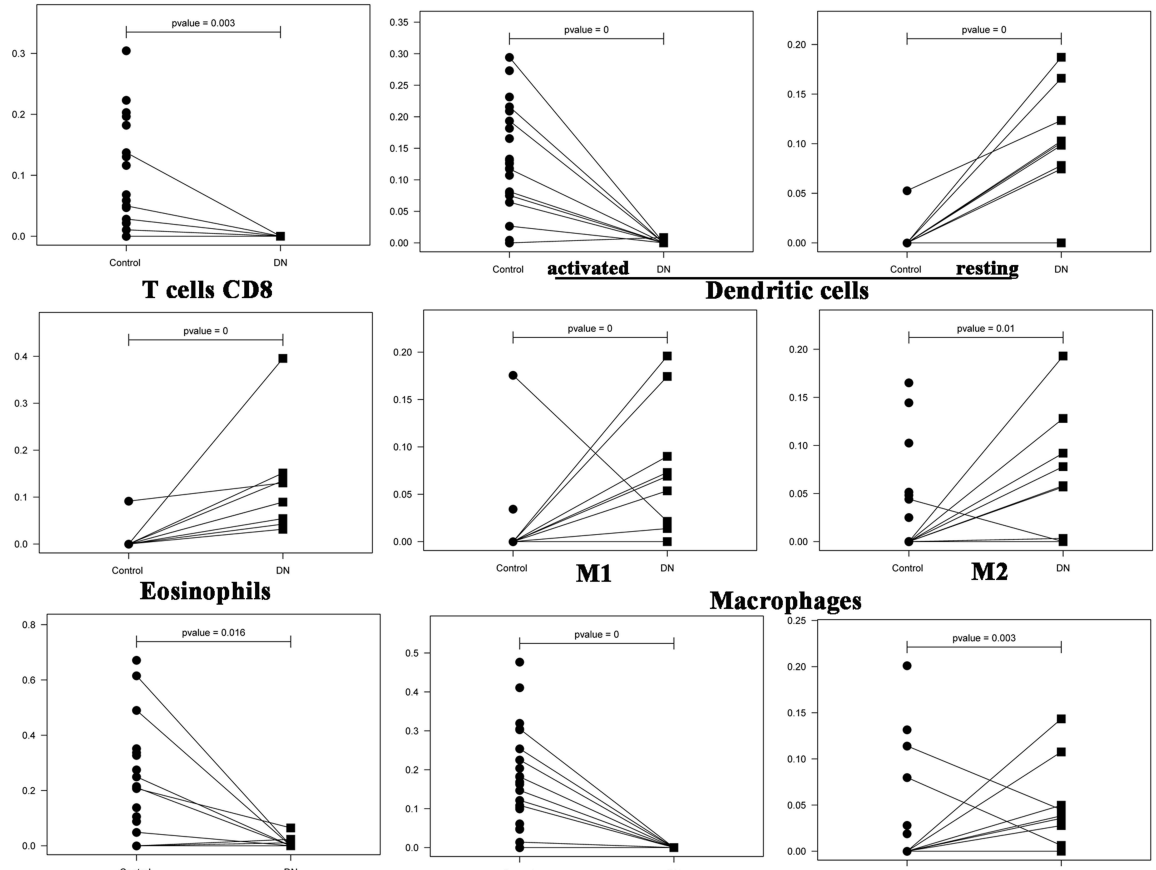

acrophages

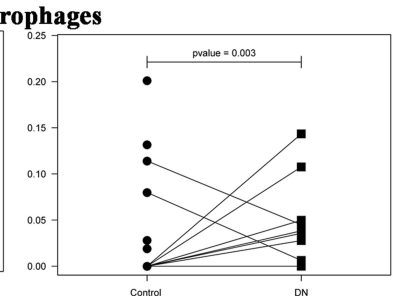

Plasma cells

Neutrophils

Mast cells activated
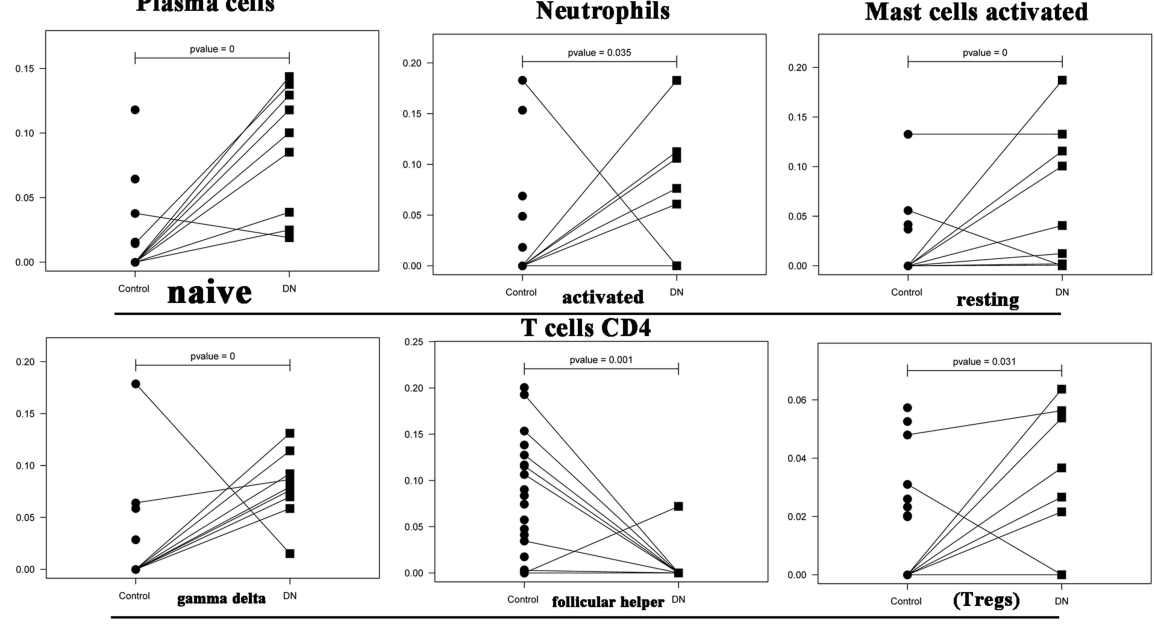

T cells

B

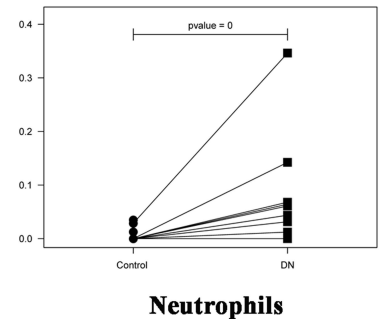

tubuli
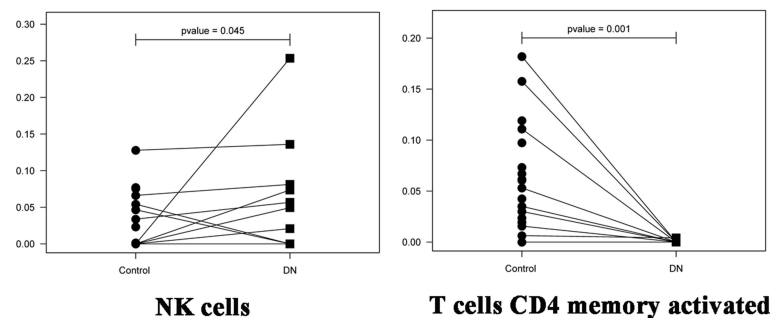

T cells CD4 memory activated

Figure 5 (A) Analysis of the relationship between hypoxia heterogeneity gene and immune cells in glomerular tissues of DN patients. (B) Analysis of the relationship between a hypoxic-heterogeneous gene in renal tubular tissues and immune cells in patients with DN. 
A

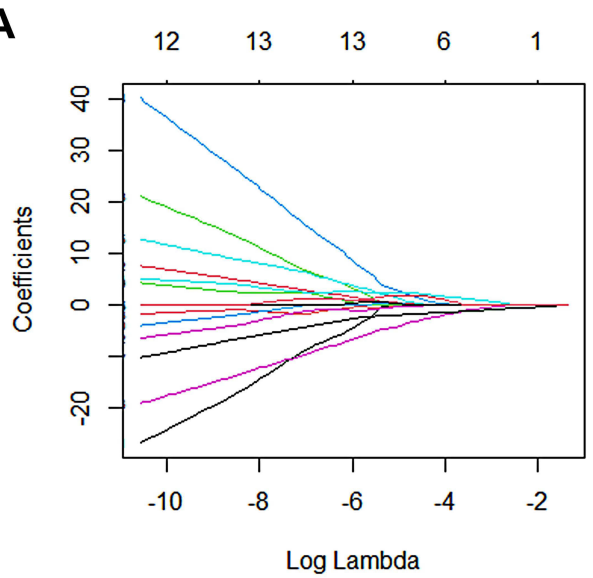

C

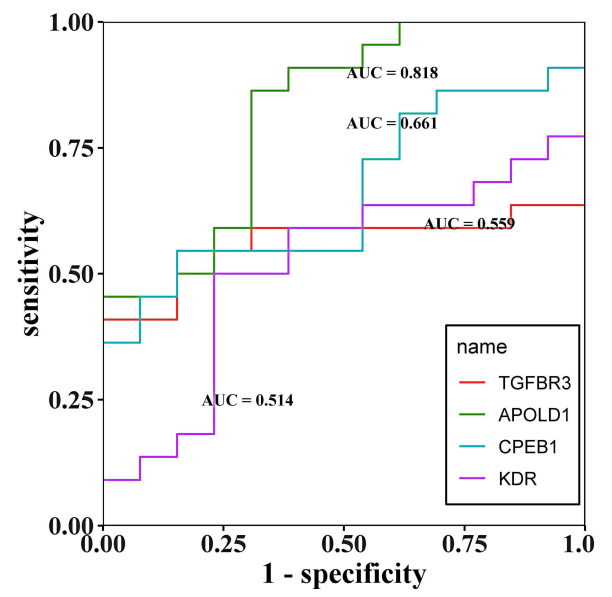

B $\begin{array}{llllllllll}12 & 12 & 13 & 13 & 13 & 11 & 6 & 4 & 1 & 1\end{array}$

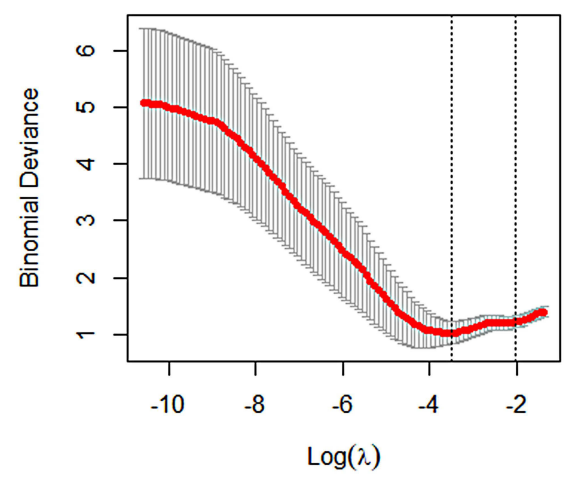

D

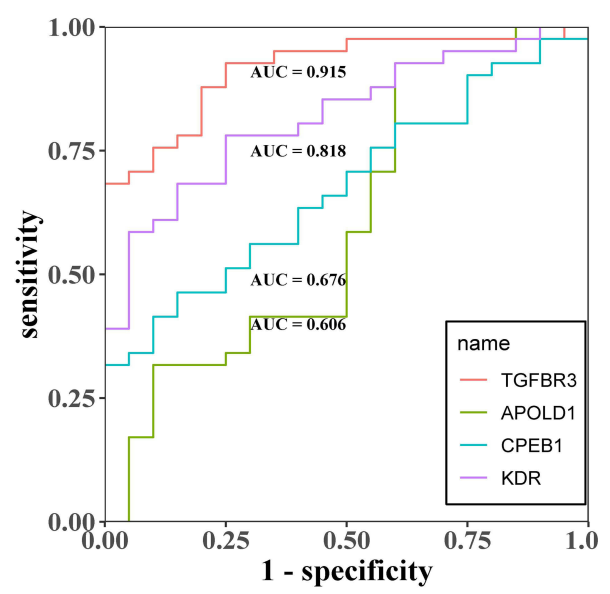

Figure 6 (A and B) Lasso regression analysis to screen the better genes for differential diagnosis of glomerular hypoxia in DN; (C) The GSE30I22 data set was used to assess the accuracy of further diagnosis of glomerular hypoxia genes in patients with DN after screening with LASSO regression; (D) The experimental set was used to assess the accuracy of further diagnosis of glomerular hypoxia genes in patients with DN after screening with LASSO regression.

CPEB1, and KDR have the potential diagnostic ability for glomerular injury in DN.

\section{Hypoxia-Associated Genes, PSMB8, PSMB9, RHOA, VCAMI, and CDKNIB, Have High Diagnostic Accuracy for DN with Tubular Injury}

To evaluate the accuracy of differential gene diagnosis of renal tubular hypoxia based on LASSO regression and ROC regression analysis, five hypoxia-associated genes, PSMB8, PSMB9, RHOA, VCAM1, and CDKN1B, were screened by LASSO regression. For the diagnostic, in the test GSE30122 dataset, the AUC values of PSMB8 (0.988), PSMB9 (0.996), RHOA (0.950), VCAM1 (0.900) and CDKN1B (0.888); besides, to validate the results of test set, GSE30529 dataset was used to evaluate the diagnostic ability of tubular injury of $\mathrm{DN}$, and it turns out that the AUC values of PSMB8 (0.992), PSMB9 (0.975), RHOA (0.950), VCAM1 (0.925) and CDKN1B (0.825); the results showed that their average diagnostic accuracy of these five genes, PSMB8 (0.990), PSMB9 (0.986), RHOA (0.950), VCAM1 (0.913) and CDKN1B (0.857), the diagnostic accuracy of these five genes is extremely high (AUC > 0.8) (Figure $8 \mathrm{~A}-\mathrm{D})$. In order to obtain more evidence to support the high diagnostic value of PSMB8, PSMB9, RHOA, CDKN1B, and VCAM1 genes in renal tubule injury in diabetic nephropathy, we normalized and clustered renal tubule transcriptomic datasets GSE30529 and GSE99340 as renal tubule transcriptomic datasets after PCA (Figure 9A and B). The diagnostic AUC values of 5 genes were verified, and the results showed that the diagnostic AUC values of PSMB8, PSMB9, RHOA, CDKN1B, and VCAM1 genes were 


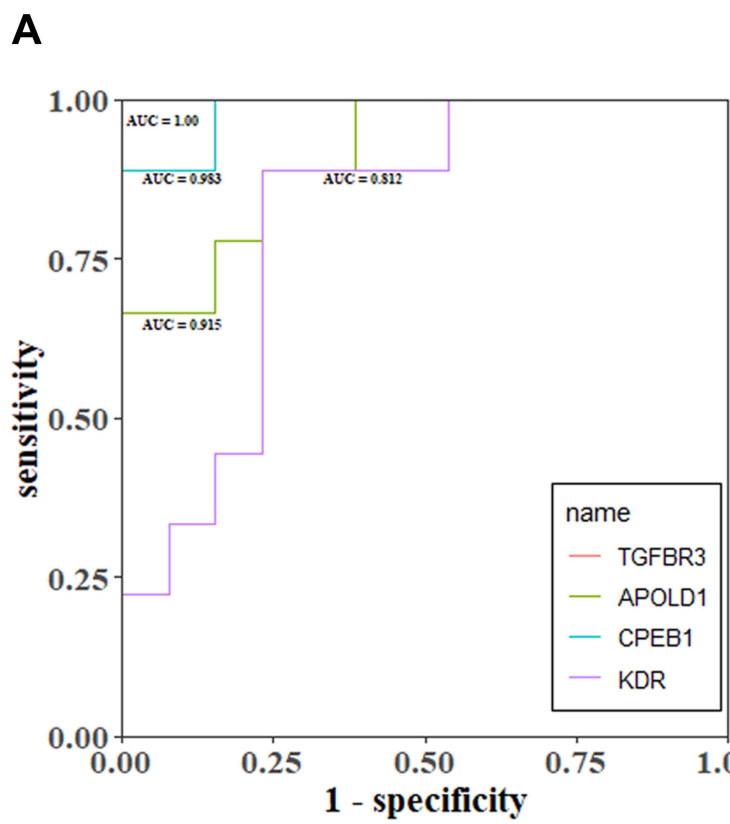

B

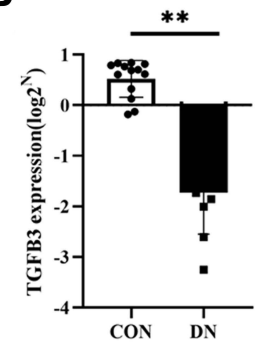

E

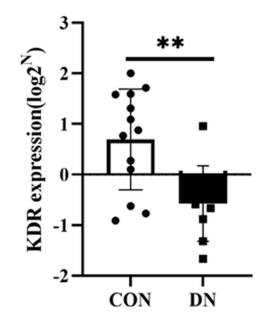

C
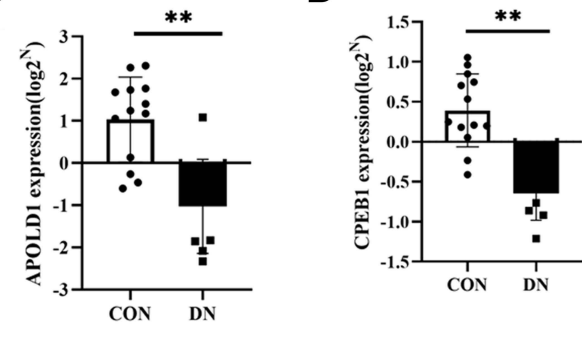

$\mathbf{F}$

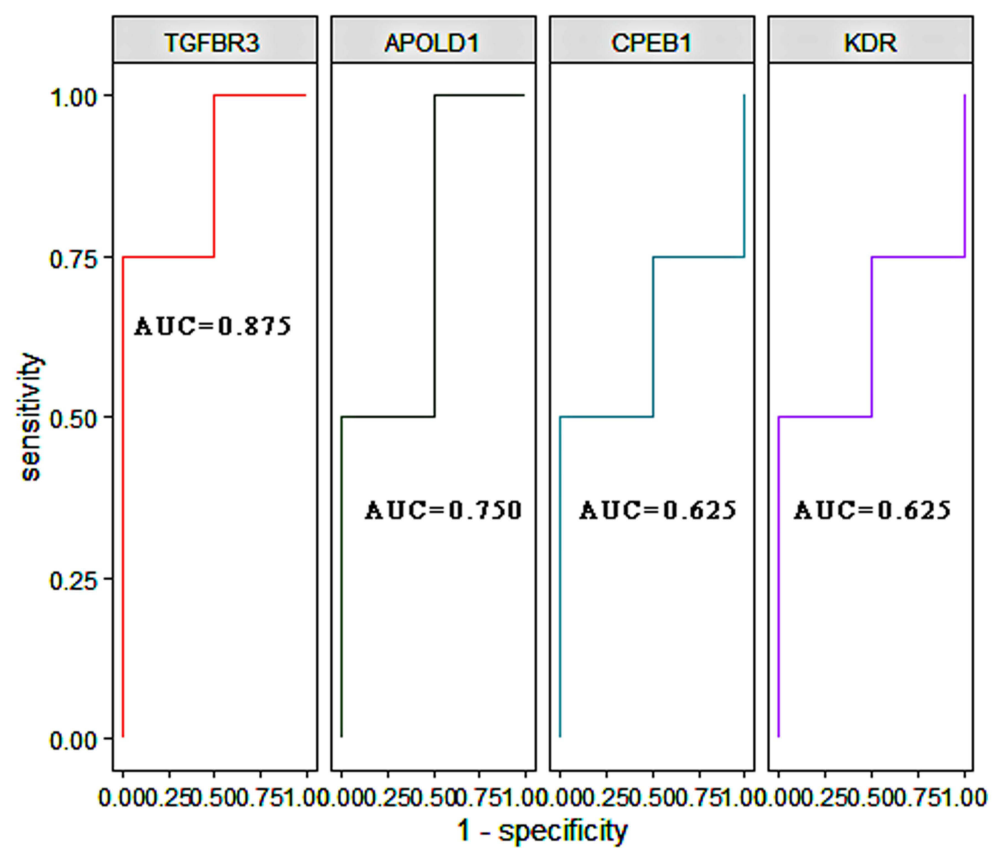

Figure 7 (A) ROC analysis of TGFBR3, APOLDI, CPEBI and KDR in glomerular transcriptome data; (B-E) Corrected transcriptome expression of TGFBR3, APOLDI, CPEBI and KDR; (F) ROC analysis of TGFBR3, APOLDI, CPEBI and KDR in podocyte transcriptome expression data. $* P<0.05 ; * * P<0.01$.

$0.827,0.821,0.780,0.768$, and 0.693 (Figure 9C), respectively. Meanwhile, all five genes were significantly upregulated in the DN group (Figure 9D-H), demonstrating high diagnostic capacity for glomerular injury. Literature indicates that mesangial cells play an irreplaceable role in the morphological and functional maintenance of renal tubules, and the loss of mesangial cells will lead to the destruction of renal tubules. We verified that PSMB8, PSMB9, RHOA, and CDKN1B gene expressions were significantly upregulated in the mesangial cell transcriptomic dataset GSE52734. However, VCAM1 gene expression was not found in this dataset (Figure 9I-L). These results further provide evidence for PSMB8, PSMB9, RHOA, and CDKN1B genes as markers of renal tubular 
injury in potential diabetic nephropathy. Whether the VCAM1 gene is not expressed in mesangial cells needs to be further explored in the future.

\section{Significant Differentially Expressed Genes in DN Can Activate Immune Cells}

Anoxic genes affect the immune state of DN after glomerular injuries, such as T cells, T cells CD8, T cell CD4 subtypes, dendritic cell, eosinophil granulocyte, macrophage subtypes, plasma cells, neutrophil, and mast cells WGCNA confirmed enrichment in the tan-colored module of hypoxia-mediated renal tubular differential gene expression data of DN (Figure 10A-D); In addition, renal tubular hypoxia was influenced by major immune cell neutrophil, T cell CD4 and natural killer cell tested by WGCNA to verify that the related genes were enriched in cyan and gray modules (Figure 10E-H).

\section{T Cell Involvement in the Regulation of} DN Glomerulopathy is Associated with the Interaction of Hypoxia-Related Genes TGFBR3, APOLDI, CPEBI, and KDR

Based on WGCNA screening the expression of TGFBR3, APOLD1, CPEB1, and KDR-related genes in glomerular tissue, the results showed that the expression of TGFBR3, APOLD1, CPEB1, and KDR-related genes in glomerular tissue could be correlated with the expression of TGFBR3 and KDR, APOLD1 and CPEB1 in glomerular tissue (Figure 11A-D). The expression of hypoxia gene KDR and TGFBR3 in turquoise module and the changes of T cell line and macrophage cell line were observed. It is suggested that KDR and TGFBR3 may be involved in the regulation of the $\mathrm{DN}$ immune response; the mechanism is related to the immune regulation of both $\mathrm{T}$ cells and macrophages (Figure 10D). Meanwhile, the T cell line and macrophage line of APOLD1 and CPEB1 also changed in the Brown module. We speculate that APOLD1 and CPEB1 may participate in the immune reaction process (Figure 10D). In order to explore the regulation mechanism of TGFBR 3 and KDR, APOLD1 and CPEB1 in turquoise module and brown module on DN immune response, Student's $t$-test $(P<0.01)$ (Figure 11A-F) was used to screen TGFBR3 and KDR in turquoise module and Brown module, the relationship between KDR and CPEB1 was analyzed statistically. The results showed that KDR could initiate the secondary regulatory network, which is mediated by NPSHI gene, and TGFBR3 can participate in BMP7 and $\mathrm{BMP} 2$, the results showed that $\mathrm{CTGF}, \mathrm{DCN}$, and $\mathrm{CPEB} 1$ were involved in the immunoregulation of $\mathrm{DN}$ by $\mathrm{T}$ cells and macrophages, the interaction of CTGF and $\mathrm{DCN}$ with MANSC1 and the interaction of CPEB1 with MAP1B, and plays a certain role (Figure 11G).

\section{T Cells are Involved in DN Regulation and are Associated with Hypoxia-Related Genes PSMB8, PSMB9, RHOA, VCAMI, and CDKNIB}

Based on WGCNA screening the gene expression of T cell line corresponding to hypoxia-associated genes PSMB8, PSMB9, RHOA, VCAM1 and CDKN1B in renal tubules (Figure 10E-H), the results showed that the expression of hypoxia gene CDKN1B and RHOA in blue module and the changes of $\mathrm{T}$ cell line and neutrophil cell line were observed, which suggested that CDKN1B and RHOA may be involved in the regulation of $\mathrm{DN}$ immune response, the mechanism is related to the immune regulation of both $\mathrm{T}$ cells and neutrophils (Figure 10G and F). The T cell line and neutrophil line of PSMB8 and PSMB9 also changed in a turquoise module. We speculate that PSMB8 and PSMB9 may participate in the immune reaction process, and the VCAM1 also plays a role in a grey model (Figure 10G and F). To explore the regulation mechanism of PSMB8, PSMB9, RHOA, VCAM1, and CDKN1B in turquoise, blue and grey modules on DN immune response. Using Student's $t$-test (Figure 12A-J) $(P<$ $0.01)$ to screen PSMB8 and PSMB9 in a turquoise module and the role of RHOA and CDKN1B in a blue module, with the VCAM1 in a grey module, the relationship between PSMB8, PSMB9, RHOA, VCAM1, and CDKN1B was analyzed statistically, and the genes with correlation regulation were selected and analyzed by PPI database. Furthermore, the genes with correlated regulation were selected for PPI analysis using the STRING database (https://www.string-db.org/). The results showed that RHOA, VCAM1, CDKN1B, PSMB8, and PSMB9 are related to each other (Figure 12K), suggesting that the anti-DN immunoregulation mechanism is used of $\mathrm{T}$ cells and centrocytes is related to the interaction of RHOA, VCAM1, CDKN1B, PSMB8, and PSMB9.

\section{Discussion}

DN is a chronic microvascular disease caused by diabetes. $^{21}$ Early diagnosis of DN is beneficial to the treatment. In this way, people will get the long-term improvement of life quality. Traditional indicators of 
A
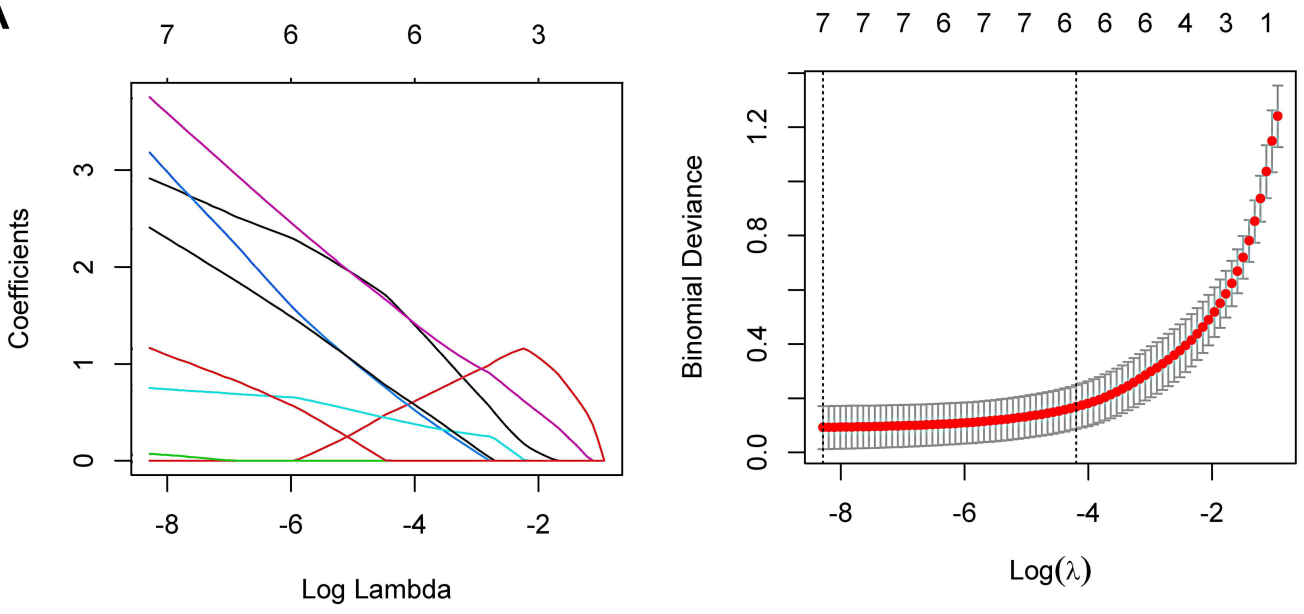

B

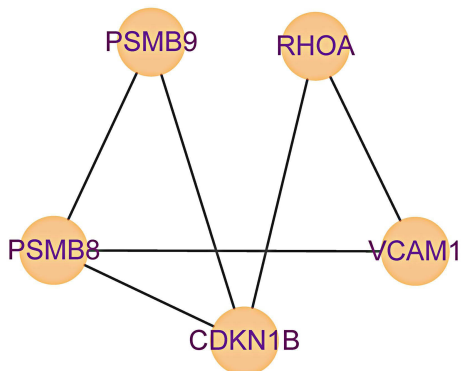

C $\quad 1.00$

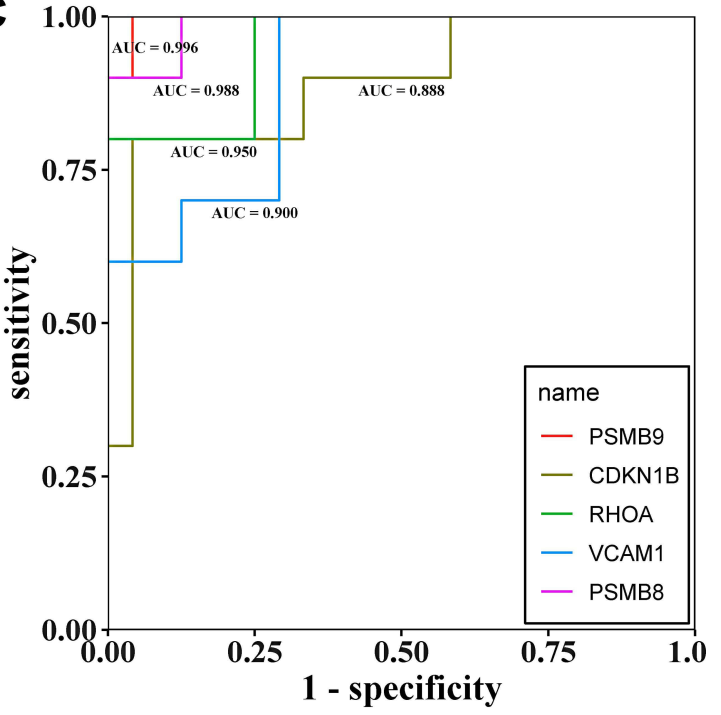

D

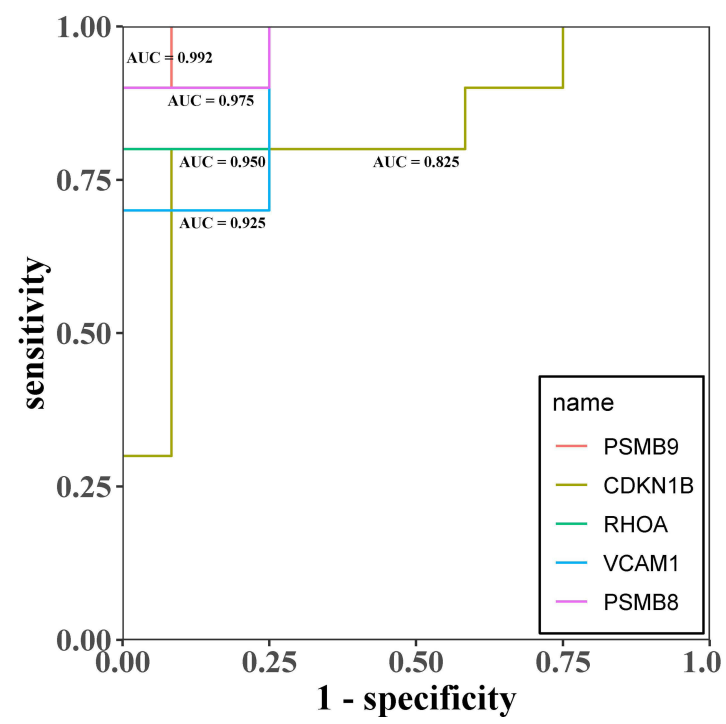

Figure 8 (A and B) Lasso regression analysis to screen the better genes for differential gene diagnosis of DN; (C) GSE30I22 data set based on the further diagnostic accuracy of the gene for tubular hypoxia in patients with DN after screening by LASSO regression. (D) GSE96804 data set based on the further diagnostic accuracy of the gene for tubular hypoxia in patients with DN after screening by LASSO regression. 


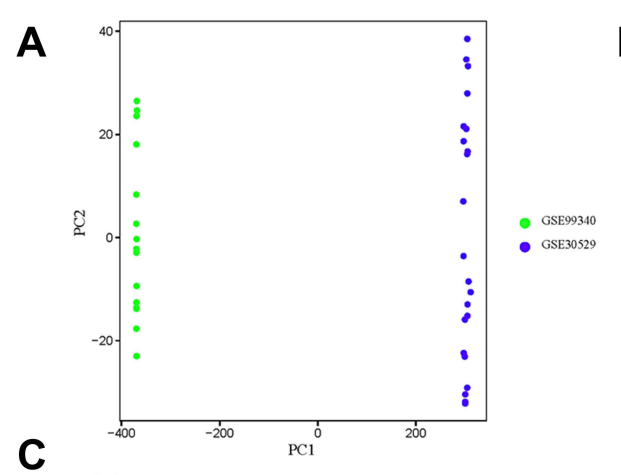

C

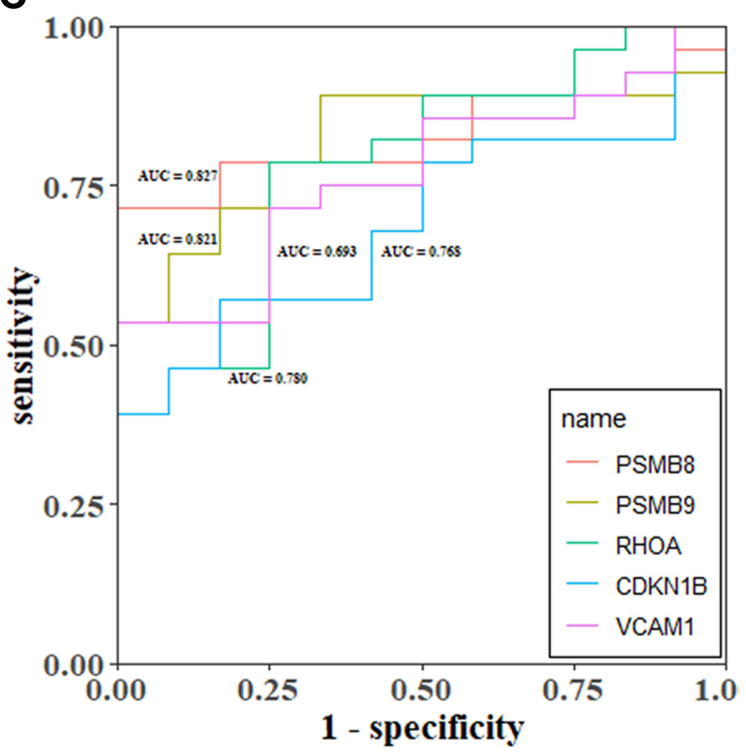

I

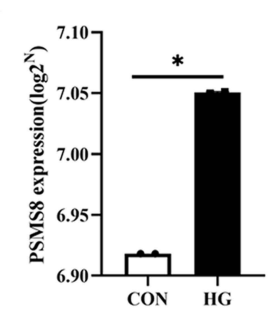

J

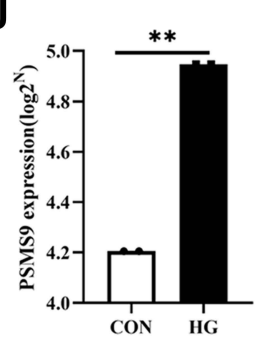

K

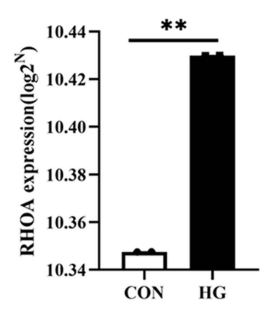

L

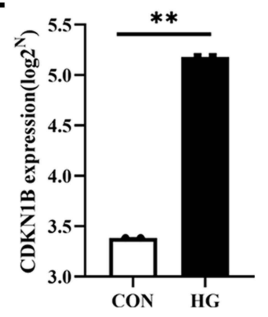

B

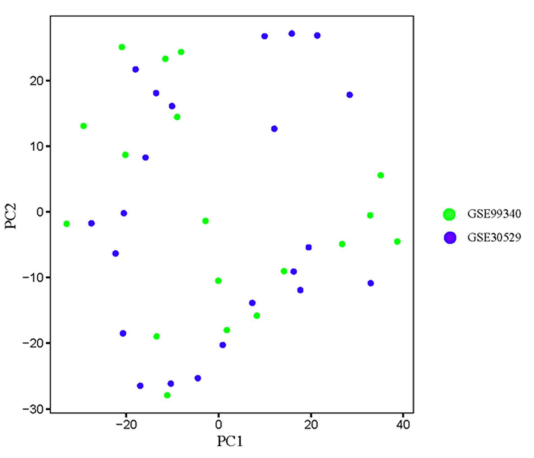

D $\quad$ E
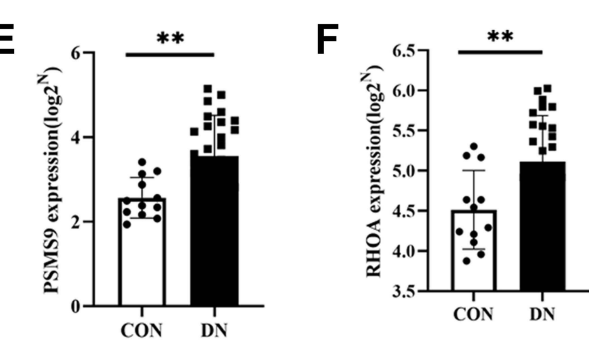

H
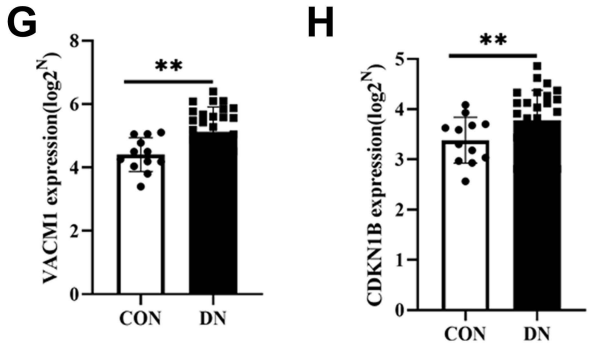

C

Figure 9 (A and B) PCA analysis of GSE99340 and GSE30529; (C) ROC analysis of PSMB8, PSMB9, RHOA, CDKNIB and VCAMI in renal tubular transcriptome data; (DH) Corrected transcriptome expression of PSMB8, PSMB9, RHOA, CDKNIB and VCAMI; (I-L) Corrected transcriptome expression of PSMB8, PSMB9, RHOA and CDKNIB in mesangial cell transcriptome expression data. $* P<0.05 ; * * P<0.01$.

renal function, such as eGFR, creatinine nitrogen levels, and 24-hour proteinuria, still play an essential role. However, some studies have pointed out that the ability to assess disease diagnosis using only renal function indicators is limited, ${ }^{22-24}$ and DN kidney injury is not limited to the glomerulus. Renal tuberosity may also occur in 
A

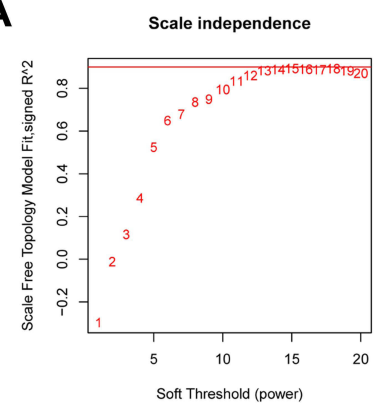

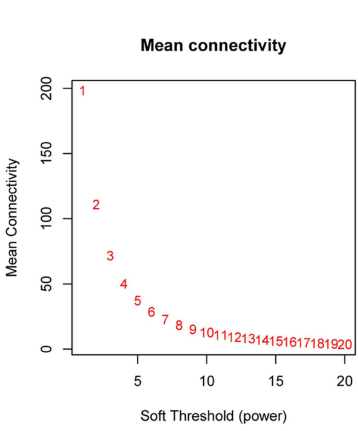

Module-trait relationships

\section{C}

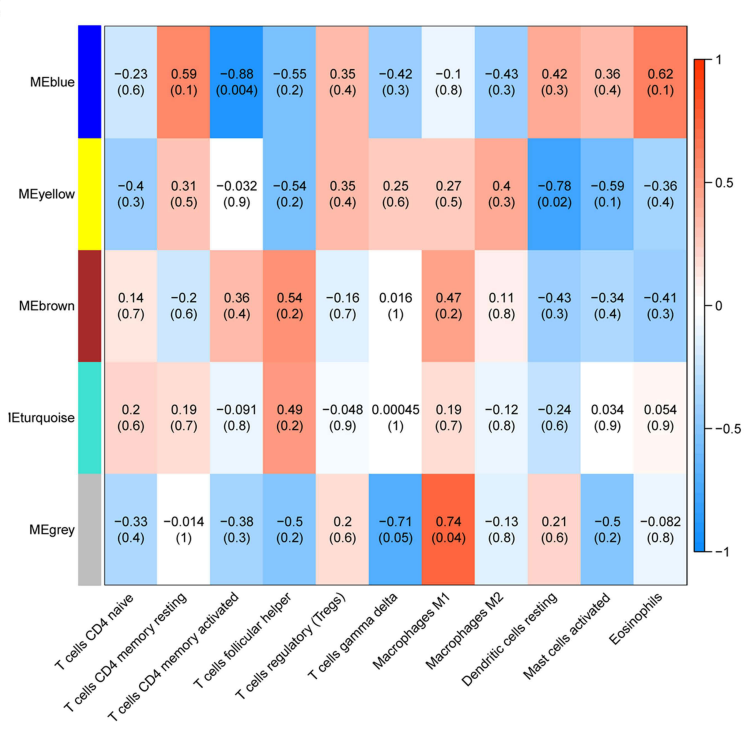

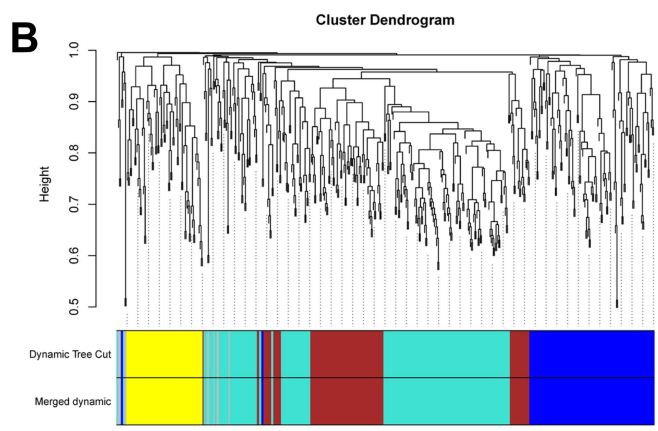

D

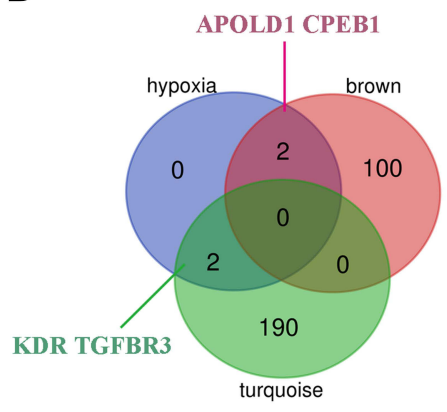

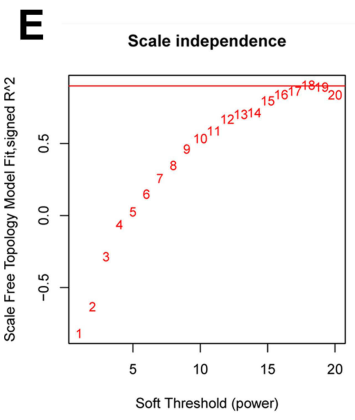

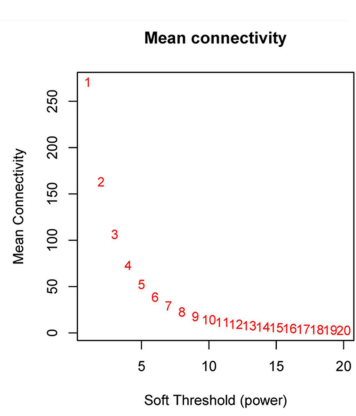

F

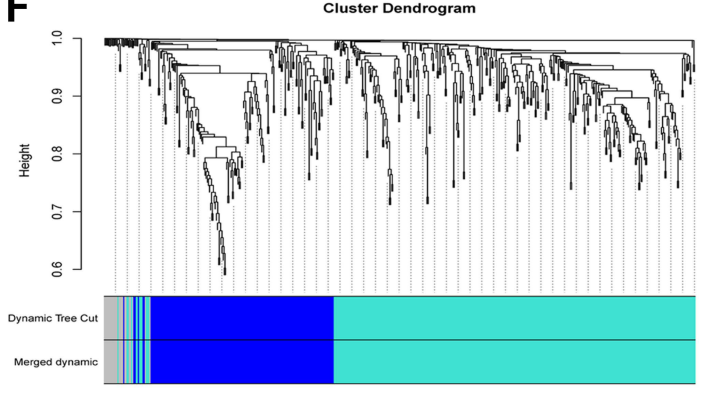

H

G

Module-trait relationships

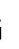

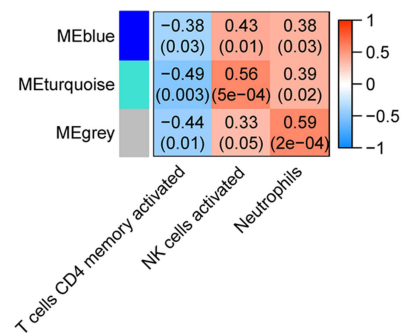

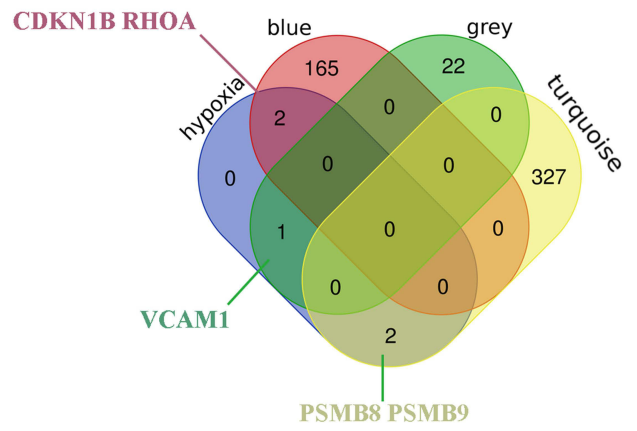

Figure 10 (A-D) Uses WGCNA to test the concentration of immune cells in glomeruli of DN; (E-H) WGCNA tests the concentration of immune cells in renal tubules of DN. 
A

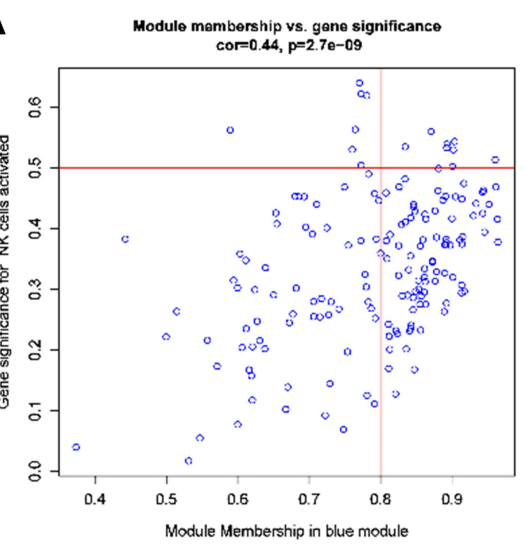

B

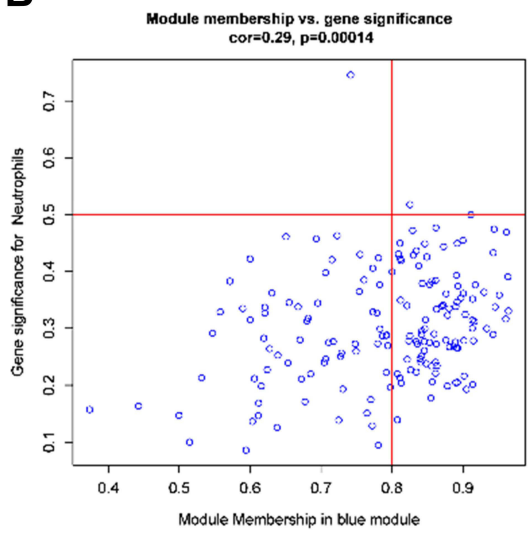

C

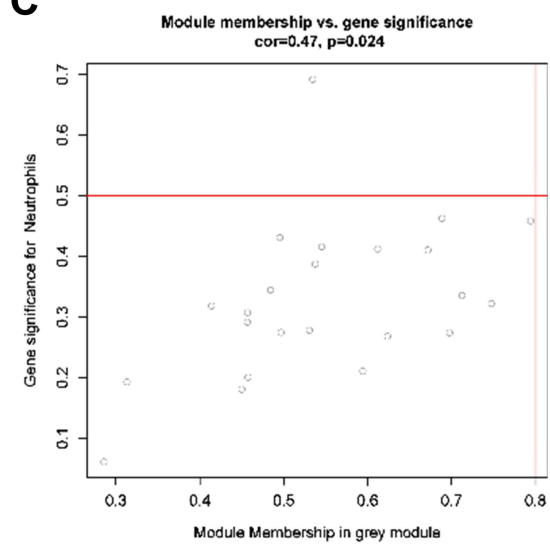

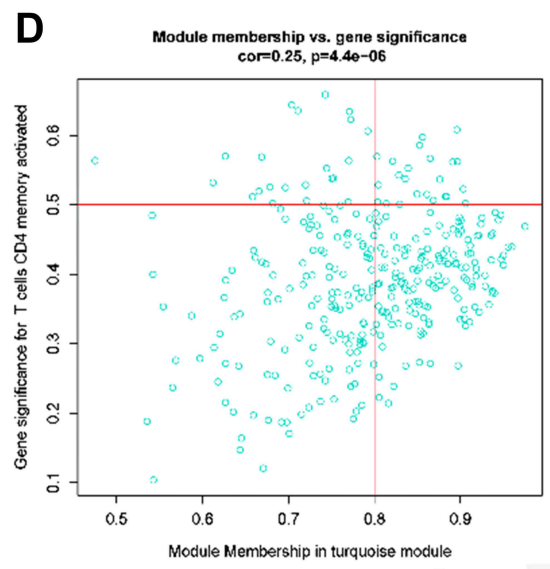

E Module membership vs. gene significance

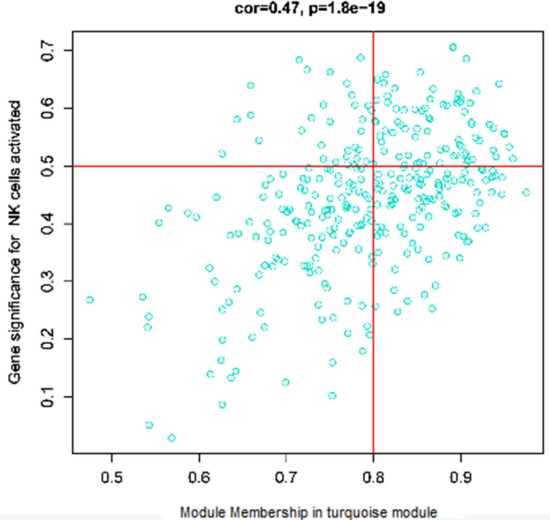

F Module membership vs. gene significance
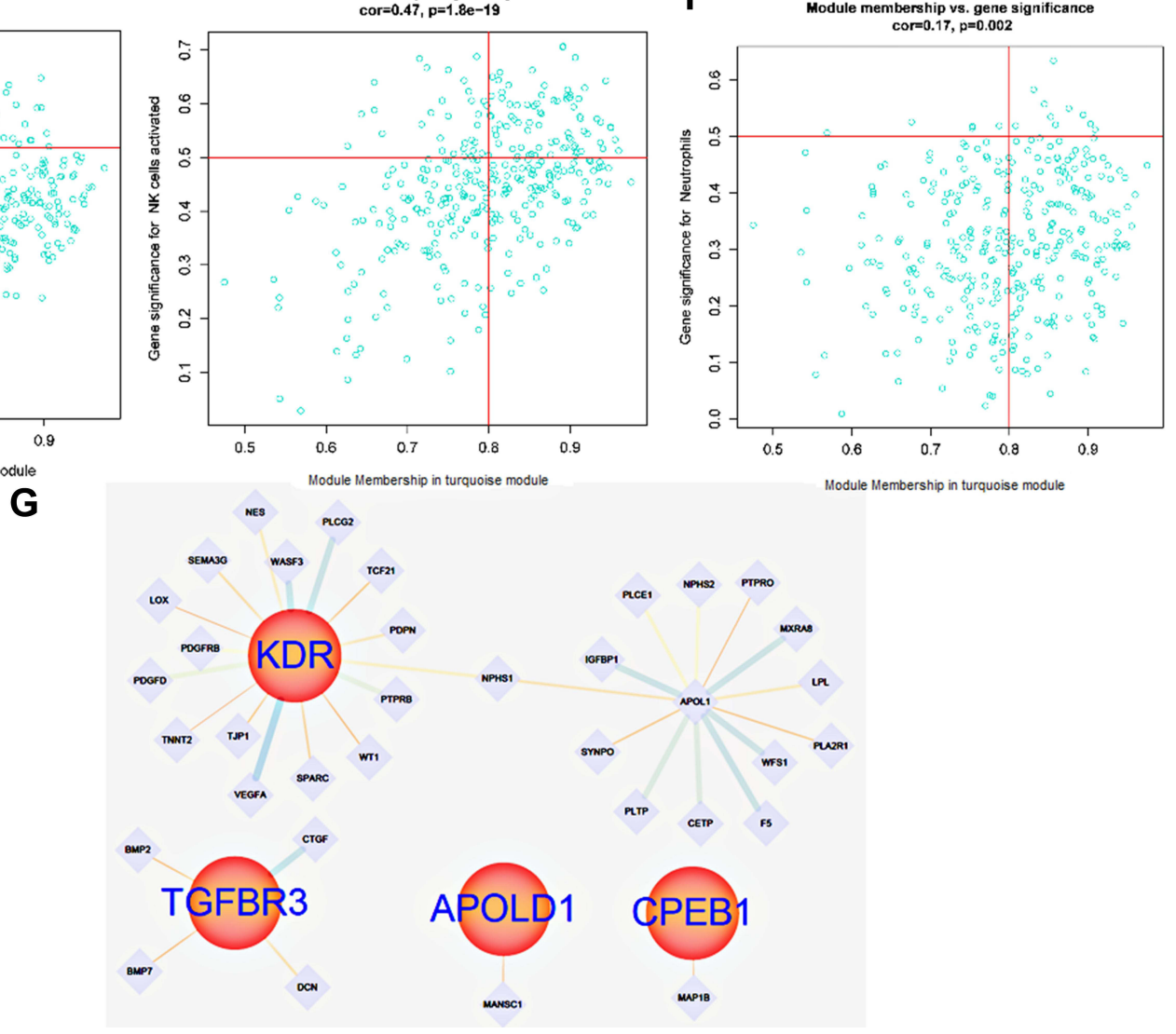

Figure II (A and B) Gene interaction of hypoxia-associated genes CDKNIB and RHOA in the blue module corresponding to T cell line; (C) Expression of hypoxiaassociated genes VCAMI in the grey module; (D-F) Gene expression of the T cell line corresponding to the cyan module of hypoxia-associated genes PSMB8 and PSMB9 in renal tubules; (G) Gene interaction of the T cell line corresponding to hypoxia-associated genes PSMB8, PSMB9, RHOA, VCAMI and CDKNIB in renal tubules.

patients with renal disease. The study found that hypoxia and immune-mediated inflammation play a role in the regulation of diabetic renal damage, of which immune cells can take part in the progress of DN, hypoxia and inflammation were involved in the immunoregulation of DN, with highly specific hypoxia-related genes could be used for the diagnosis of DN.
It has been found that the immune ability of diabetic patients is weak, among which is the function of $\mathrm{T}$ lymphocyte. Immunoglobulin and NK cell activity is generally decreased, and there are both cellular and humoral immune deficiency. ${ }^{25,26} \mathrm{DN}$ is a chronic vascular response secondary to diabetes. $\mathrm{T}$ cells play a significant role in immune regulation, of which Th1 cells can be 

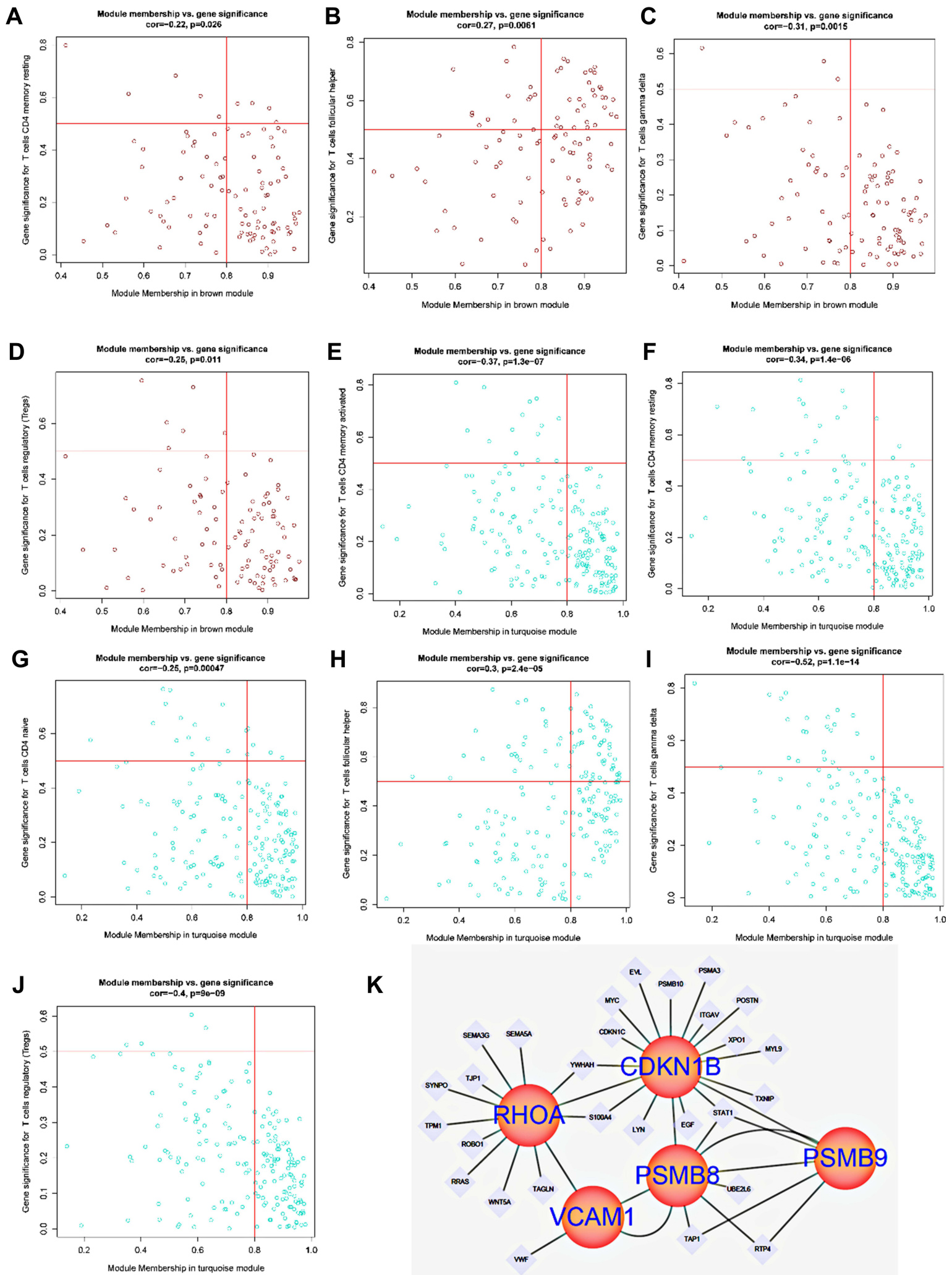

Figure 12 (A-D) Gene interaction of cell line corresponding to the brown module in the hypoxia-associated genes APOLDI and CPEBI; (E-J) Expression of TGFBR3 and KDR-related T cell lines in the glomerulus, (K) Interaction of TGFBR3 and KDR related T cell lines in the glomerulus. 
induced to form macrophage M1. It can secrete TNF- $\alpha$, IL-1 $\beta$, and IL-12 to promote the inflammatory reaction of Th1 cells. ${ }^{27}$ Moreover, Treg cells are an essential subgroup of $\mathrm{T}$ cells, which can regulate the activity of various immune cells and protect normal tissues and organs from immune injury. ${ }^{28}$ According to the site of formation. Treg cells are innate regulatory $\mathrm{T}$ cells derived from the initial $\mathrm{T}$ cells and induced $\mathrm{T}$ cells derived from peripheral blood. TGF- $\beta$ maintained at high concentrations inhibits the secretion of inflammatory cytokines (INF- $\gamma$ and IL-2) and promotes the differentiation of initial $\mathrm{T}$ cells into Tregs. In this study, the cell model predicted the downregulation of Tregs and Th2, up-regulation of Th1 cells and NKT and antigen-presenting cells, further analysis of the glomerular and tubular immunocyte phenotypes by the CIBERSORT model revealed an up-regulation of M1 expression in macrophages after glomerulopathy, which indicates that glomerular lesions are most likely to differentiate with Th1 cells to form macrophage M1 after glomerulopathy. Besides, the activation of antigen-presenting cells was inhibited after glomerulopathy, and the activation of neutrophil cells remained downregulated.

In contrast, Tregs cells' activation was unregulated, which suggested that the activation of Th1 cells in glomerular disease is also inhibited by Tregs cells at a moderate level. Above the renal tubular disease, neutrophil and natural killer cell play a significant role. In conclusion, when DN occurs, macrophages, $\mathrm{T}$ cells, and neutrophils, the degree of dependence of the glomerulus and tubules on immune cells is different, which is related to the inflammatory response regulated by $\mathrm{T}$ cells to a great extent.

To investigate the mechanism of $\mathrm{T}$ cell action, this study explored the mechanism of a GSE30122 data set, 519 differentially expressed genes were associated with renal tubular injury, and 493 differentially expressed genes were associated with glomerulus, based on the UniProt database, 16 hypoxia genes were found to be associated with glomerular disease by WGCNA analysis, which was mainly enriched in the HIF1 signaling pathway, and 17 hypoxia genes were associated with renal tubular disease, which was enriched in a phagocytic signaling pathway.

Hypoxia gene was further identified by combined analysis of lasso and ROC regression showing that after the glomerular injury, the AUC values of TGFBR3, APOID1, CPEB1, and KDR were 0.737, 0.712, 0.669 and 0.666. There is considerable clinical evidence that inflammation is a critical factor in the initiation and development of DN. ${ }^{29-31}$ TGF- $\beta 1$ and TGF- $\beta 2$ are the two subtypes of TGF- $\beta$. It finds that TGFBR3 can specifically bind to these two subtypes. As mentioned above, when TGF- $\beta$ secretion is reduced, Th1 cells can be induced by cytokines to produce macrophage M1, which can secrete TNF$\alpha$, IL- $1 \beta$, and IL-12 to promote the inflammatory response of Th1 cells. In the DN model of glomerulus and proximal tubular epithelial cells, increased expression of Th1 cells was found. Through NF- $\kappa B$ signal transduction, TNF- $\alpha$ induces the transcription of cytokinin, thus affecting cell survival, proliferation, and adhesion, promoting inflammation and apoptosis. ${ }^{32,33}$ Apolipoprotein $\mathrm{L}$ domain is a protein encoded by the APOLD1 gene in the human body. As an early endothelial reaction protein, APOL1 harbors three coding-sequence variants discovered in kidney disease patients of African ancestry and linked to nondiabetic chronic kidney disease (CKD) ${ }^{34,35}$ Thus, the APOLD1 gene may play a role in regulating endothelial cell signal and vascular function. What's more, DN is a chronic vascular disease, and all of this, the APOPRLD gene can regulate vascular signal, which indicates that the APOPRLD gene is a potential diagnostic gene of DN. Besides, the study reveals that the HIF-1 signaling pathway was activated following glomerular injury induced by diabetes. Research shows that overexpression of CPEB1 inhibits the mRNA translation of HIF- $1 \alpha$ and thus downregulates the expression of HIF-1 $1 \alpha^{36}$ Furthermore, the above mentioned Th1 cells can generate macrophage M1 under the induction of cytokines to further activate the inflammatory reaction of Th1 cells, ${ }^{37,38}$ overexpression of CPEB1 can inhibit the expression of HIF-1 $\alpha$, so it is inferred that CPEB1 can mediate the expression of HIF-1 $\alpha$ and slow down the inflammatory response of Th1 cells. An interesting manifestation of this study is that KDR, which has mitotic and chemotactic activity, plays a significant role in vascular permeability and angiogenesis, ${ }^{39}$ there may be a link between HIF-1 $\alpha$ and KDR.

Immune hypoxia genes, PSMB8, PSMB9, RHOA, VCAM1, and CDKN1B, are involved in regulating $\mathrm{T}$ cells after the renal tubular lesion. Five genes, PSMB8 (0.990), PSMB9 (0.986), RHOA (0.950), VCAM1 (0.913) and CDKN1B (0.857), had higher diagnostic ability (AUC $>0.7$ ). PPI analysis showed that PSMB8, PSMB9, RHOA, VCAM1, and CDKN1B had potential interaction. Vascular cell adhesion molecule-1 (VCAM1) is a member of the Immunoglobulin superfamily. VCAM-1 increased in the infiltrating cells of endothelial cells and renal 
tubulointerstitial cells in experimental DN; its level was positively correlated with the degree of proteinuria. PSMB8, PSMB9, and PSMB10 form a complex that activates the immune response. Recent studies have shown that the PSMB8 immunoproteasome can degrade NF- $\mathrm{BB}$ production inhibition of $\mathrm{NF}-\kappa \mathrm{B}$ production by $\mathrm{NF}-\kappa \mathrm{B}$ inhibitors, ${ }^{40}$ which are a key step in inflammation regulation. In contrast, PSMB 8 can activate NF- $\kappa B$ through the action of the immunoproteasome and then activate the transcription of inflammation-related factors in the cell nucleus, thus promoting the development of DN inflammation. Ras homolog gene family member (RhoA) plays an essential role in the cytoskeleton, maintaining cell morphology, cell migration, and smooth muscle cell contraction. ${ }^{41}$ It also plays a key role in many biological activities, such as smooth muscle cell proliferation, cell adhesion, platelet aggregation, contact inhibition, growth, and apoptosis; ${ }^{42}$ RhoA /ROCK signaling pathway is coinvolved with its downstream effector Rho-associated protein kinase (ROCK), which has been shown to activate when exposed to diabetic kidney cells, ${ }^{43}$ some studies have found that FASUDIL treatment inhibits RhoA/ ROCK activation and NF- $\kappa \mathrm{B}$ nuclear translocation significantly reduces the levels of renal cell adhesion molecule-1 and TGF- $\beta 1$ proteins ${ }^{44}$ and attenuates NF$\kappa \mathrm{B}$-inflammatory gene upregulation-mediated $\mathrm{DN}$ progression. $^{45,46}$ CDKN1B, also known as cyclindependent kinase inhibitor 1B (P27, KIP1), is highly diagnostic in this study of renal tubular injury caused by diabetes, and G1 cells are now thought to be associated with growth inhibition or hypertrophy, cell cycle transitions can be blocked by the cyclin-dependent kinase reactive protein/kinase inhibitor (CIP/Kip) family of cyclindependent kinase inhibitors (CKIS: P21, P27, and P57). P27 and P21 were expressed in hypertrophic renal tubular epithelial cells. ${ }^{47,48}$ Therefore, it can be concluded that P27 inhibits cell cycle transition after DN, leading to renal tubular epithelial cells remaining in the G1 phase and cell and growth inhibition. These shreds of evidence suggest that inflammation and immune response are involved in the regulation of renal tubular injury and have an excellent diagnostic ability for renal tubular injury induced by diabetes. The authors conclude, due to sample size limitations and publication bias in open data sets, to a certain extent, we plan to carry out experiments in the follow-up study to verify the conclusions of this paper, to prove the reliability of the forecast results, to make up for the limitations of this study.

\section{Conclusion}

DN is a complicated chronic vascular inflammation, and the diagnostic markers of glomerular and renal tubular injury are different. TGFBR3, APOLD1, CPEB1, and KDR, are highly specific to the glomerulus, and the other genes, PSMB8, PSMB9, RHOA, VCAM1, and CDKN1B, are highly diagnostic for renal tubules. Lesions mainly involving $\mathrm{T}$ cells and neutrophil responses may be associated with hypoxia and activation of inflammatory responses.

\section{Ethics Statement}

In this manuscript, we used GSE30122, GSE96804, GSE46897, ${ }^{49}$ GSE52734, ${ }^{50}$ GSE99340, ${ }^{51}$ GSE30528 ${ }^{17}$ and GSE30529 ${ }^{17}$ transcribed data from the GEO public database (https://www.ncbi.nlm.nih/GEO/). Patients in the database have been ethically approved. Users can download the data for research and publication free of charge. Our research is based on open-source data, so there are no ethical issues or conflicts of interest. The First Affiliated Hospital of Kunming Medical University gave scientists free access to the patient data set included in this study from the NCBI GEO database for use in scientific research. The data set included in the GEO data platform already has an ethical review statement. Therefore, the articles using open data for the study do not involve the application of patient information and sample database in the GEO data platform, besides, the First Affiliated Hospital of Kunming Medical University Ethics Committee has given this manuscript an ethical exemption.

\section{Acknowledgments}

This work was supported by a grant from National Natural Science Foundation of China (Project Number: 82160142), Yunnan Health Technical Training Project of High-level Talents (Project Number: D-2018027) and Yunnan Basic Research Projects - General Program (Project Number: 2019FB092).

\section{Author Contributions}

All authors made a significant contribution to the work reported, whether that is in the conception, study design, execution, acquisition of data, analysis and interpretation, or in all these areas; took part in drafting, revising or critically reviewing the article; gave final approval of the version to be published; have agreed on the journal to which the article has been submitted; and agree to be accountable for all aspects of the work. 


\section{Disclosure}

The authors report no conflicts of interest in this work.

\section{References}

1. Koay Y, Tan G, Phang S, et al. A phase IIb randomized controlled trial investigating the effects of tocotrienol-rich vitamin $\mathrm{E}$ on diabetic kidney disease. Nutrients. 2021;13(1):258. doi:10.3390/nu13010258

2. Wende AR, Schell JC, Ha C-M, et al. Maintaining myocardial glucose utilization in diabetic cardiomyopathy accelerates mitochondrial dysfunction. Diabetes. 2020;69(10):2094-2111. doi:10.2337/db19-1057

3. Lord T, Nixon B. Metabolic changes accompanying spermatogonial stem cell differentiation. Dev Cell. 2020;52(4):399-411. doi:10.1016/ j.devcel.2020.01.014

4. Srivastava A, Tomar B, Prajapati S, Gaikwad AB, Mulay SR. Advanced non-invasive diagnostic techniques for visualization and estimation of kidney fibrosis. Drug Discov Today. 2021;26 (8):2053-2063. doi:10.1016/j.drudis.2021.02.016

5. de Bhailís ÁM, Chrysochou C, Kalra PA. Inflammation and oxidative damage in ischaemic renal disease. Antioxidants. 2021;10(6):845. doi:10.3390/antiox 10060845

6. Spencer S, Wheeler-Jones C, Elliott J. Hypoxia and chronic kidney disease: possible mechanisms, therapeutic targets, and relevance to cats. Vet J. 2021;274:105714. doi:10.1016/j.tvj1.2021.105714

7. Scholz H, Boivin FJ, Schmidt-Ott KM, et al. Kidney physiology and susceptibility to acute kidney injury: implications for renoprotection. Nat Rev Nephrol. 2021;17(5):335-349. doi:10.1038/s41581-021-00394-7

8. Tang SC, Yiu WH. Innate immunity in diabetic kidney disease. Nat Rev Nephrol. 2020;16(4):206-222. doi:10.1038/s41581-019-0234-4

9. Lee H, Fessler MB, Qu P, Heymann J, Kopp JB. Macrophage polarization in innate immune responses contributing to pathogenesis of chronic kidney disease. BMC Nephrol. 2020;21(1):1-13. doi:10.1186/ s12882-020-01921-7

10. Herrmann SM, Perazella MA. Immune checkpoint inhibitors and immune-related adverse renal events. Kidney Int Rep. 2020;5 (8):1139-1148. doi:10.1016/j.ekir.2020.04.018

11. Tong X, Yu Q, Ankawi G, Pang B, Yang B, Yang H. Insights into the role of renal biopsy in patients with T2DM: a literature review of global renal biopsy results. Diabetes Ther. 2020;1-17. doi:10.1007/ s13300-020-00888-w

12. Sun L, Shang J, Xiao J, Zhao Z. Development and validation of a predictive model for end-stage renal disease risk in patients with diabetic nephropathy confirmed by renal biopsy. PeerJ. 2020;8: e8499. doi:10.7717/peerj.8499

13. Selby NM, Taal MW. An updated overview of diabetic nephropathy: diagnosis, prognosis, treatment goals and latest guidelines. Diabetes Obes Metab. 2020;22:3-15. doi:10.1111/dom.14007

14. Kim HR, Na KR, Lee JI, et al. Progression to chronic kidney disease according to albuminuria in diabetic nephropathy patients with preserved renal function. 2020.

15. Ihara K, Skupien J, Kobayashi H, et al. Erratum. profibrotic circulating proteins and risk of early progressive renal decline in patients with type 2 diabetes with and without albuminuria. Diabetes Care. 2021;44(2):631. doi:10.2337/dc20-0630

16. Li L, Jick S, Breitenstein S, Michel A. Prevalence of diabetes and diabetic nephropathy in a large US commercially insured pediatric population, 2002-2013. Diabetes Care. 2016;39(2):278-284. doi: $10.2337 / \mathrm{dc} 15-1710$

17. Woroniecka KI, Park ASD, Mohtat D, Thomas DB, Pullman JM, Susztak K. Transcriptome analysis of human diabetic kidney disease. Diabetes. 2011;60(9):2354-2369. doi:10.2337/db10-1181

18. Pan Y, Jiang S, Hou Q, et al. Dissection of glomerular transcriptional profile in patients with diabetic nephropathy: SRGAP2a protects podocyte structure and function. Diabetes. 2018;67(4):717-730. doi: $10.2337 / \mathrm{db} 17-0755$
19. Fan Y, Yi Z, D'Agati VD, et al. Comparison of kidney transcriptomic profiles of early and advanced diabetic nephropathy reveals potential new mechanisms for disease progression. Diabetes. 2019;68 (12):2301-2314. doi:10.2337/db19-0204

20. Trendafilov N, Gallo M. Principal Component Analysis (PCA). Multivariate Data Analysis on Matrix Manifolds. Springer; 2021:89-139. doi:10.1007/978-1-4419-9863-7_1276

21. Lim AK, Tesch GH. Inflammation in diabetic nephropathy. Mediators Inflamm. 2012;2012:146154. doi:10.1155/2012/146154

22. Blank M, Thompson A, Hausner E, Rouse R. Biomarkers of drug-induced acute kidney injury: a regulatory perspective. Expert Opin Drug Metab Toxicol. 2018;14(9):929-936. doi:10.1080/ 17425255.2018.1511701

23. Chawla L, Bellomo R, Bihorac A, et al. Acute kidney disease and renal recovery: consensus report of the Acute Disease Quality Initiative (ADQI) 16 workgroup. Nat Rev Nephrol. 2017;13 (4):241-257. doi:10.1038/nrneph.2017.2

24. Thomas M, Blaine C, Dawnay A, et al. The definition of acute kidney injury and its use in practice. Kidney Int. 2015;87(1):62-73. doi:10.1038/ki.2014.328

25. Askenasy N. Mechanisms of autoimmunity in the non-obese diabetic mouse: effector/regulatory cell equilibrium during peak inflammation. Immunology. 2016;147(4):377-388. doi:10.1111/ imm. 12581

26. Zheng Z, Zheng F. Immune cells and inflammation in diabetic nephropathy. J Diabetes Res. 2016;2016:1841690. doi:10.1155/ 2016/1841690

27. Ma W, Gao F, Gu K, Chen D. The role of monocytes and macrophages in autoimmune diseases: a comprehensive review. Front Immunol. 2019;10:1140. doi:10.3389/fimmu.2019.01140

28. Bayati F, Mohammadi M, Valadi M, Jamshidi S, Foma A, SharifPaghaleh E. The therapeutic potential of regulatory T cells: challenges and opportunities. Front Immunol. 2020;11:585819. doi:10.3389/fimmu.2020.585819

29. Li S, Jia Y, Xue M, et al. Inhibiting Rab27a in renal tubular epithelial cells attenuates the inflammation of diabetic kidney disease through the miR-26a-5p/CHAC1/NF-kB pathway. Life Sci. 2020;261:118347. doi:10.1016/j.lfs.2020.118347

30. Salti T, Khazim K, Haddad R, Campisi-Pinto S, Bar-Sela G, Cohen I. Glucose induces IL-1 $\alpha$-dependent inflammation and extracellular matrix proteins expression and deposition in renal tubular epithelial cells in diabetic kidney disease. Front Immunol. 2020;11:1270. doi:10.3389/fimmu.2020.01270

31. Zhou Y, Ma X, Han J, et al. Metformin regulates inflammation and fibrosis in diabetic kidney disease through TNC/TLR4/NF- $\mathrm{kB} / \mathrm{miR}-$ 155-5p inflammatory loop. World J Diabetes. 2021;12(1):19-46. doi:10.4239/wjd.v12.i1.19

32. Yang M, Zhang Y, Ren J. Acetylation in cardiovascular diseases: molecular mechanisms and clinical implications. Biochimica Et Biophysica Acta Mol Basis Dis. 2020;1866(10):165836. doi:10.1016/j.bbadis.2020.165836

33. Zouhal H, Zare-Kookandeh N, Haghighi M, et al. Physical activity and adipokine levels in individuals with type 2 diabetes: a literature review and practical applications. Rev Endocr Metab Disord. 2021. doi:10.1007/s11154-021-09657-x

34. Hu C, Klopfer E, Ray P. Human apolipoprotein L1 (ApoL1) in cancer and chronic kidney disease. FEBS Lett. 2012;586(7):947-955. doi:10.1016/j.febslet.2012.03.002

35. Neumann C, Silver D, Venkateshwari V, et al. MBOAT7-driven phosphatidylinositol remodeling promotes the progression of clear cell renal carcinoma. Mol Metabol. 2020;34:136-145. doi:10.1016/j. molmet.2020.01.011

36. Chen P, Huang Y. CPEB2-eEF2 interaction impedes HIF-1 $\alpha$ RNA translation. EMBO J. 2012;31(4):959-971. doi:10.1038/ emboj.2011.448 
37. Christopoulos P, Gjølberg T, Krüger S, Haraldsen G, Andersen J, Sundlisæter E. Targeting the notch signaling pathway in chronic inflammatory diseases. Front Immunol. 2021;12:668207. doi:10.3389/fimmu.2021.668207

38. Mills C, Kincaid K, Alt J, Heilman M, Hill A. M-1/M-2 macrophages and the Th1/Th2 paradigm. J Immunol. 2000;164(12):6166-6173. doi:10.4049/jimmunol.164.12.6166

39. De Falco S. The discovery of placenta growth factor and its biological activity. Exp Mol Med. 2012;44(1):19. doi:10.3858/emm.2012.44.1.025

40. Carney E. Proteasome impairment can lead to CKD. Nat Rev Nephrol. 2021;17(4):222. doi:10.1038/s41581-021-00409-3

41. Jones M, Zha J, Humphries M. Connections between the cell cycle, cell adhesion and the cytoskeleton. Biol Sci. 2019;374 (1779):20180227. doi:10.1098/rstb.2018.0227

42. Kilian L, Voran J, Frank D, Rangrez A. RhoA: a dubious molecule in cardiac pathophysiology. J Biomed Sci. 2021;28(1):33. doi:10.1186/ s12929-021-00730-w

43. Neves K, Harvey A, Moreton F, et al. ER stress and Rho kinase activation underlie the vasculopathy of CADASIL. JCI Insight. 2019;4(23). doi:10.1172/jci.insight.131344

44. Wang E, Tu W, Do D, et al. Benzo(a)pyrene enhanced dermatophagoides group 1 (Der $\mathrm{f} 1$ )-induced TGF $\beta 1$ signaling activation through the aryl hydrocarbon receptor-rhoa axis in asthma. Front Immunol. 2021;12:643260. doi:10.3389/fimmu.2021.643260

45. Komers R. Rho kinase inhibition in diabetic kidney disease. Br J Clin Pharmacol. 2013;76(4):551-559. doi:10.1111/bcp.12196
46. Matoba K, Kawanami D, Okada R, et al. Rho-kinase inhibition prevents the progression of diabetic nephropathy by downregulating hypoxia-inducible factor 1 $\alpha$. Kidney Int. 2013;84(3):545-554. doi:10.1038/ki.2013.130

47. Sinha S, Dwivedi N, Woodgett J, et al. Glycogen synthase kinase-3 $\beta$ inhibits tubular regeneration in acute kidney injury by a FoxM1dependent mechanism. FASEB J. 2020;34(10):13597-13608. doi:10.1096/fj.202000526RR

48. Zhang Y, Wang W, Su S, et al. Roles of 12-lipoxygenase and its interaction with angiotensin II on p21 and p27 expression in diabetic nephropathy. Nephron. 2019;142(1):61-70. doi:10.1159/000496440

49. Merscher-Gomez S, Guzman J, Pedigo CE, et al. Cyclodextrin protects podocytes in diabetic kidney disease. Diabetes. 2013;62 (11):3817-3827. doi:10.2337/db13-0399

50. Iwata Y, Furuichi K, Hashimoto S, et al. Pro-inflammatory/Th1 gene expression shift in high glucose stimulated mesangial cells and tubular epithelial cells. Biochem Biophys Res Commun. 2014;443 (3):969-974. doi:10.1016/j.bbrc.2013.12.072

51. Shved N, Warsow G, Eichinger F, et al. Transcriptome-based network analysis reveals renal cell type-specific dysregulation of hypoxia-associated transcripts. Sci Rep. 2017;7(1):8576. doi:10.1038/s41598-017-08492-y
Journal of Inflammation Research

\section{Publish your work in this journal}

The Journal of Inflammation Research is an international, peerreviewed open-access journal that welcomes laboratory and clinical findings on the molecular basis, cell biology and pharmacology of inflammation including original research, reviews, symposium reports, hypothesis formation and commentaries on: acute/chronic inflammation; mediators of inflammation; cellular processes; molecular mechanisms; pharmacology and novel anti-inflammatory drugs; clinical conditions involving inflammation. The manuscript management system is completely online and includes a very quick and fair peerreview system. Visit http://www.dovepress.com/testimonials.php to read real quotes from published authors. 